\title{
Searching for $Z^{\prime}$ bosons at the $\mathrm{P} 2$ experiment
}

\author{
P.S. Bhupal Dev, ${ }^{a}$ Werner Rodejohann, ${ }^{b}$ Xun-Jie $\mathbf{X} \mathbf{u}^{c}$ and Yongchao Zhang ${ }^{d, a}$ \\ ${ }^{a}$ Department of Physics and McDonnell Center for the Space Sciences, Washington University, \\ St. Louis, MO 63130, U.S.A. \\ ${ }^{b}$ Max-Planck-Institut für Kernphysik, \\ Saupfercheckweg 1, 69117 Heidelberg, Germany \\ c Service de Physique Théorique, Université Libre de Bruxelles, \\ Boulevard du Triomphe, CP225, 1050 Brussels, Belgium \\ ${ }^{d}$ School of Physics, Southeast University, \\ Nanjing 211189, China \\ E-mail: bdev@wustl.edu, werner.rodejohann@mpi-hd.mpg.de, \\ xunjie.xu@ulb.ac.be, zhangyongchao@seu.edu.cn
}

ABSTRACT: The P2 experiment aims at high-precision measurements of the parity-violating asymmetry in elastic electron-proton and electron- ${ }^{12} \mathrm{C}$ scatterings with longitudinally polarized electrons. We discuss here the sensitivity of P2 to new physics mediated by an additional neutral gauge boson $Z^{\prime}$ of a new $\mathrm{U}(1)^{\prime}$ gauge symmetry. If the charge assignment of the $\mathrm{U}(1)^{\prime}$ is chiral, i.e., left- and right-handed fermions have different charges under $\mathrm{U}(1)^{\prime}$, additional parity-violation is induced directly. On the other hand, if the $\mathrm{U}(1)^{\prime}$ has a non-chiral charge assignment, additional parity-violation can be induced via mass or kinetic $Z-Z^{\prime}$ mixing. By comparing the $\mathrm{P} 2$ sensitivity to existing constraints, we show that in both cases $\mathrm{P} 2$ has discovery potential over a wide range of $Z^{\prime}$ mass. In particular, for chiral models, the P2 experiment can probe gauge couplings at the order of $10^{-5}$ when the $Z^{\prime}$ boson is light, and heavy $Z^{\prime}$ bosons up to $79(90) \mathrm{TeV}$ in the proton $\left({ }^{12} \mathrm{C}\right)$ mode. For non-chiral models with mass mixing, the $\mathrm{P} 2$ experiment is sensitive to mass mixing angles smaller than roughly $10^{-4}$, depending on model details and gauge coupling magnitude.

KeYwords: Beyond Standard Model, Gauge Symmetry

ArXiv EPrint: 2103.09067 


\section{Contents}

1 Introduction 1

2 Parity-violating asymmetry in elastic electron scattering 3

3 New physics effects $\quad 6$

3.1 Chiral U(1)' models 6

3.2 Non-chiral $\mathrm{U}(1)^{\prime}$ models and mixing-induced couplings 8

$\begin{array}{lll}4 & \text { P2 sensitivity study } & 10\end{array}$

$\begin{array}{lll}4.1 \text { Method } & 10\end{array}$

$\begin{array}{lll}4.2 & \text { Current experimental limits } & 12\end{array}$

$\begin{array}{lll}4.3 & \text { P2 sensitivities } & 15\end{array}$

$\begin{array}{lll}\text { 4.3.1 Chiral models } & 15\end{array}$

$\begin{array}{lll}4.3 .2 & \text { Non-chiral models } & 19\end{array}$

5 Conclusion $\quad 24$

$\begin{array}{ll}\text { A The contribution of the axial-vector coupling } & 28\end{array}$

\section{Introduction}

Why parity is violated in elementary particle interactions remains one of the biggest mysteries in physics. Within the Standard Model (SM) of particle physics, parity-violation is caused by the weak $Z$ and $W^{ \pm}$bosons, which couple differently to left- and right-handed fermions. This chiral charge assignment of the SM fermions leads to various interesting phenomena and precision tests of the SM [1]. At the same time, given the fact that some form of beyond the SM (BSM) physics is expected on general grounds, an interesting question is whether the new BSM interactions are parity-conserving or parity-violating. Either way, BSM physics can influence parity-violating observables in reactions in which it participates. Thus, parity-violating searches provide an excellent avenue to probe BSM physics; see ref. [2] for a recent review.

A classic parity-violating observable can be obtained from the scattering of polarized electrons off unpolarized targets, yielding cross-sections $\sigma_{R}$ and $\sigma_{L}$ for right- and lefthanded electrons, respectively. The parity-violating left-right asymmetry, defined as

$$
A^{\mathrm{PV}}=\frac{\sigma_{R}-\sigma_{L}}{\sigma_{R}+\sigma_{L}}
$$

is then a very useful probe of parity-violation; see ref. [3] for a review. The asymmetry $A^{\mathrm{PV}}$ has been (will be) measured with high precision in low-energy polarized electron 
scattering processes [4], such as Møller scattering $e^{-} e^{-} \rightarrow e^{-} e^{-}[5,6]([7])$, as well as electron-proton [8-12] ([13]), electron-deuteron [14-19], electron- ${ }^{4} \mathrm{He}$ [20], electron- ${ }^{9} \mathrm{Be}[21]$, electron- ${ }^{12} \mathrm{C}[22]$ ([13]) and electron- ${ }^{208} \mathrm{~Pb}[23]$ scatterings. In addition, there are precise measurements of atomic parity violation (APV) using ${ }^{133} \mathrm{Cs}[24-27],{ }^{205} \mathrm{Tl}[28,29]$, ${ }^{208} \mathrm{~Pb}$ [30], ${ }^{209} \mathrm{Bi}$ [31] and ${ }^{100,102,104,106} \mathrm{Yb}$ [32]. Parity-violating asymmetries have also been measured at the high-energy colliders such as LEP, SLC, Tevatron and LHC [1]. The interplay of these various parity-violating measurements with new physics have been discussed, e.g. in refs. [33-48].

This paper deals with probing new physics using the measurements of the parityasymmetry in elastic electron-proton or electron- ${ }^{12} \mathrm{C}$ scatterings in the proposed P2 experiment at the upcoming Mainz Energy-recovering Superconducting Accelerator (MESA) facility [13]. The goal of P2, with start of data-taking expected in 2024, is to measure the parity-violating asymmetries for polarized electrons scattering off unpolarized protons or ${ }^{12} \mathrm{C}$ nuclei using a $155 \mathrm{MeV}$ electron beam, where the relative uncertainties $\Delta A^{\mathrm{PV}} / A^{\mathrm{PV}}$ are expected to be $1.4 \%$ and $0.3 \%$ respectively [13]. Such precise measurements at low momentum transfer provide not only an important test of the SM, but also a sensitive probe of BSM physics.

One natural scenario of new physics is a (light) $Z^{\prime}$ boson that couples differently to left- and right-handed SM fermions. The $Z^{\prime}$ boson will mediate new Feynman diagrams for electron scattering off proton or nucleus (see the bottom two panels in figure 1). We specifically examine how new physics in the form of a new neutral gauge boson $Z^{\prime}$ can be constrained in the P2 experiment. Depending on the origin of the parity-violation in the $Z^{\prime}$ couplings, the $\mathrm{U}(1)^{\prime}$ models accommodating the $Z^{\prime}$ gauge boson can be classified into two categories:

- Chiral theories in which left- and right-handed particles have different charge assignments under the $\mathrm{U}(1)^{\prime}$. They give a direct contribution to the parity-violating asymmetry. Some anomaly-free $\mathrm{U}(1)^{\prime}$ examples are given in table 2 .

- Vector-like or non-chiral theories in which left- and right-handed particles have identical charge assignments under the $\mathrm{U}(1)^{\prime}$. They give an indirect contribution to the parity-violating asymmetry if the $Z^{\prime}$ mixes with the SM $Z$ boson. In this paper, we will first consider a generic $\mathrm{U}(1)^{\prime}$ model with either mass mixing $\sin \theta$ or kinetic mixing $\epsilon$ in the limit of the new gauge coupling $g^{\prime} \rightarrow 0$, and then generalize to the $\mathrm{U}(1)_{B}$ and $\mathrm{U}(1)_{B-L}$ models (here $B$ and $L$ denote the baryon and lepton number, respectively), with three benchmark values of $g^{\prime} / \sin \theta=0.01,1$ and 10, and $\epsilon=0$.

Our results, shown in figures 2 to 6 , demonstrate that the $\mathrm{P} 2$ prospects of the $\mathrm{U}(1)^{\prime}$ models are rather model-dependent. However, even if all existing constraints are taken into consideration, the P2 experiment can still probe a wide range of $Z^{\prime}$ masses. For the three chiral models considered in this paper, the $\mathrm{P} 2$ experiment can probe gauge couplings down to $g^{\prime} \sim 10^{-5}$ when the $Z^{\prime}$ boson is light, as summarized in table 4 . When the $Z^{\prime}$ boson mass $m_{Z^{\prime}}$ is large, the P2 experiment probes an effective cutoff scale $\Lambda=m_{Z^{\prime}} / g^{\prime}$, which for $m_{Z^{\prime}}$ can go up to $79 \mathrm{TeV}$ in the $e+p$ mode, and even up to $90 \mathrm{TeV}$ in the $e+{ }^{12} \mathrm{C}$ mode 
(setting the $g^{\prime}$ to be the perturbative limit of $4 \pi$ ), which is well beyond direct searches at past and current high-energy colliders. For the non-chiral models, if there is only $Z-Z^{\prime}$ mass mixing, in the limit of $g^{\prime} Q^{\prime} \rightarrow 0$, the $\mathrm{P} 2$ prospects in the $e+p$ mode have been precluded by APV measurements, while P2 can probe unexplored mass mixing angles in the range of $1.1 \times 10^{-4}<\sin \theta<0.15$ in $e+{ }^{12} \mathrm{C}$ scattering, as shown in figure 3 and table 5 . If there is only kinetic mixing, the $Z^{\prime}$ boson behaves essentially like a dark photon when it is light, and it is also severely constrained when it is heavy. Such particles are easily accessible and often searched for. Therefore, the P2 prospects of the kinetic mixing angle $\epsilon$ have been precluded by existing limits, as presented in figure 4 . For illustration, we further apply our analyses to $\mathrm{U}(1)_{B}$ and $\mathrm{U}(1)_{B-L}$ models with $g^{\prime} / \sin \theta$ fixed at some benchmark values. We find that when the gauge coupling $g^{\prime}$ is sizable compared to the mass mixing angle $\sin \theta$, the $\mathrm{P} 2$ sensitivity to $\sin \theta$ can be significantly improved. Our study also shows that whether $e+p$ or $e+{ }^{12} \mathrm{C}$ scattering gives better limits depends largely on the model.

The rest of the paper is organized as follows: in section 2 we discuss general aspects of the parity-violating asymmetry, before discussing various chiral and non-chiral models that modify the parity-violating asymmetry in section 3. A sensitivity study is performed in section 4: with the procedure for obtaining the sensitivities given in section 2 , all relevant existing limits are collected in section 4.2, and the P2 sensitivities are obtained in section 4.3. The conclusions are presented in section 5. More details of the axial-vector couplings of $Z^{\prime}$ boson to proton and ${ }^{12} \mathrm{C}$ are provided in appendix A.

\section{$2 \quad$ Parity-violating asymmetry in elastic electron scattering}

In this section, we derive the parity-violating asymmetry $A^{\mathrm{PV}}$ defined in eq. (1.1) for elastic electron-proton or electron-nucleus scattering. In the SM, the leading-order contribution can be computed by evaluating the first two diagrams in figure 1 . Parity-violation enters via the $Z$ boson contribution and its interference with the parity-conserving photon diagram.

A new neutral gauge boson $Z^{\prime}$ could also contribute to $A^{\mathrm{PV}}$ either directly or indirectly via mixing with the SM $Z$ boson, depending on whether the $Z^{\prime}$ couplings to $e_{L}$ and $e_{R}$ are different or not, respectively. This is illustrated by the diagrams in the bottom two panels of figure 1 . In the mixing case, we need to canonicalize kinetic terms or diagonalize mass terms so that both $Z$ and $Z^{\prime}$ are mass eigenstates, leading to effective parity-violating couplings of the $Z^{\prime}$. Besides, in the presence of $Z-Z^{\prime}$ mixing, the $Z$ couplings may also deviate from the SM values. This is discussed in detail in section 3. In general, we can consider the following Lagrangian, which contains the neutral current (NC) interactions of the SM $Z$ boson and the most general interactions of a $Z^{\prime}$ with polarized electrons and a target nucleus $N$ :

$$
\begin{aligned}
\mathcal{L} \supset & Z_{\mu}\left(g_{e_{L}} \overline{e_{L}} \gamma^{\mu} e_{L}+g_{e_{R}} \overline{e_{R}} \gamma^{\mu} e_{R}\right)+Z_{\mu}\left(g_{V} \bar{N} \gamma^{\mu} N+g_{A} \bar{N} \gamma^{\mu} \gamma^{5} N\right) \\
& +Z_{\mu}^{\prime}\left(g_{e_{L}}^{\prime} \overline{e_{L}} \gamma^{\mu} e_{L}+g_{e_{R}}^{\prime} \overline{e_{R}} \gamma^{\mu} e_{R}\right)+Z_{\mu}^{\prime}\left(g_{V}^{\prime} \bar{N} \gamma^{\mu} N+g_{A}^{\prime} \bar{N} \gamma^{\mu} \gamma^{5} N\right) .
\end{aligned}
$$

Here $Z$ and $Z^{\prime}$ are mass eigenstates with masses denoted by $m_{Z}$ and $m_{Z^{\prime}}, g_{e_{L}}$ and $g_{e_{R}}\left(g_{e_{L}}^{\prime}\right.$ and $\left.g_{e_{R}}^{\prime}\right)$ are the effective couplings of $Z\left(Z^{\prime}\right)$ to $e_{L}$ and $e_{R}, g_{V}$ and $g_{A}\left(g_{V}^{\prime}\right.$ and $\left.g_{A}^{\prime}\right)$ are 


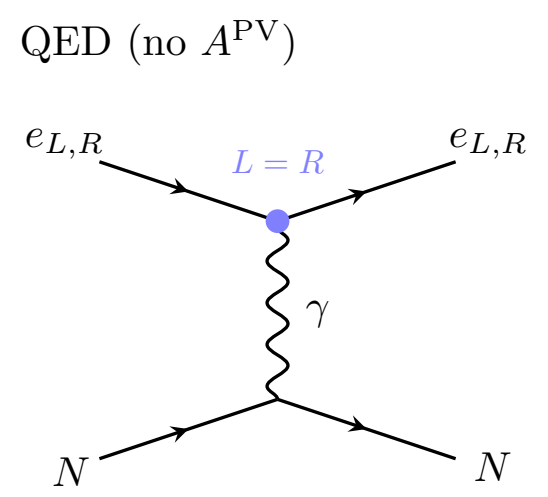

$A^{\mathrm{PV}}$ from chiral $U(1)^{\prime}$

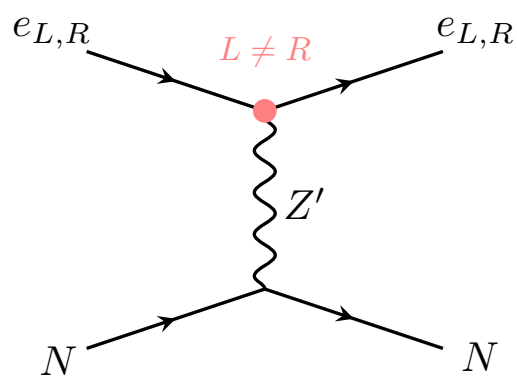

$A^{\mathrm{PV}}$ from $\mathrm{SM}$

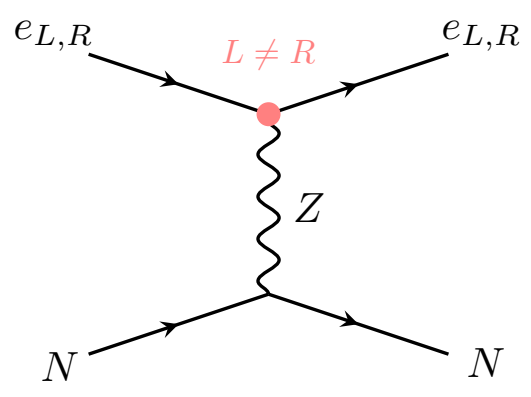

$A^{\mathrm{PV}}$ from non-chiral $U(1)^{\prime}$

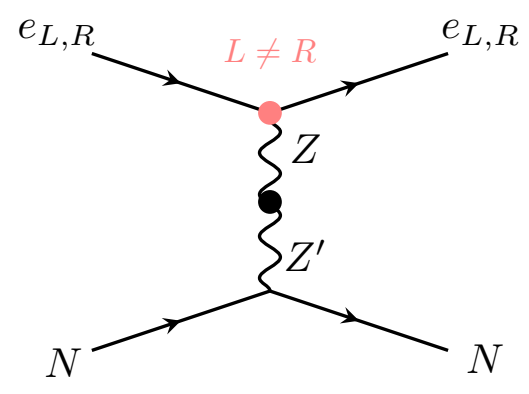

Figure 1. Leading-order processes for $A^{\mathrm{PV}}$ in the $\mathrm{SM}$ (top panels) and in the $Z^{\prime}$ models considered here (bottom panels). $N$ denotes either a proton or a nucleus $\left({ }^{12} \mathrm{C}\right.$ in the context of P2).

vector and axial-vector couplings of $Z\left(Z^{\prime}\right)$ to a nucleus, respectively. The vector couplings can be obtained by simply adding up the fundamental couplings to quarks in a nucleus with $\mathcal{N}$ neutrons and $\mathcal{Z}$ protons as follows:

$$
\begin{aligned}
& g_{V}=\mathcal{Z}\left(g_{u_{L}}+g_{u_{R}}+\frac{1}{2} g_{d_{L}}+\frac{1}{2} g_{d_{R}}\right)+\mathcal{N}\left(g_{d_{L}}+g_{d_{R}}+\frac{1}{2} g_{u_{L}}+\frac{1}{2} g_{u_{R}}\right) \\
& g_{V}^{\prime}=\mathcal{Z}\left(g_{u_{L}}^{\prime}+g_{u_{R}}^{\prime}+\frac{1}{2} g_{d_{L}}^{\prime}+\frac{1}{2} g_{d_{R}}^{\prime}\right)+\mathcal{N}\left(g_{d_{L}}^{\prime}+g_{d_{R}}^{\prime}+\frac{1}{2} g_{u_{L}}^{\prime}+\frac{1}{2} g_{u_{R}}^{\prime}\right)
\end{aligned}
$$

Here the couplings to chiral quarks $\left(g_{u_{L}}, g_{u_{R}}, g_{d_{R}}^{\prime}\right.$, etc.) are defined in a way similar to $g_{e_{L}}$ and $g_{e_{R}}$ in eq. (2.1). In table 1 we list the SM values of these couplings as well as new physics values which will be derived later in section 3 .

As for the axial-vector couplings $\left(g_{A}\right.$ and $\left.g_{A}^{\prime}\right)$, albeit not calculable from first principles, their contributions to $A^{\mathrm{PV}}$ are suppressed by electron energy over target mass, as we show in appendix A. If we therefore ignore the contribution of $g_{A}$ and $g_{A}^{\prime}$, the amplitude of $e_{L, R}^{-}+N \rightarrow e_{L, R}^{-}+N$ reads

$$
i \mathcal{M}_{L, R} \propto\left[\overline{u_{3}} \gamma^{\mu} P_{L, R} u_{1}\right]\left[\overline{u_{4}} \gamma_{\mu} G_{L, R} u_{2}\right]
$$




\begin{tabular}{|c|c|c|c|c|}
\hline models & SM & chiral models & non-chiral model & \\
\hline$A^{\mathrm{PV}}$ caused by & $\mathrm{NC}$ & $Q_{e_{L}}^{\prime} \neq Q_{e_{R}}^{\prime}$ & $\epsilon B^{\mu \nu} F_{\mu \nu}^{\prime}$ & $\delta m^{2} Z^{\mu} Z_{\mu}^{\prime}$ \\
\hline$g_{e_{L}}$ & $g_{Z}\left(-\frac{1}{2}+s_{W}^{2}\right)$ & $g_{e_{L}}^{\mathrm{SM}}$ & $g_{e_{L}}^{\mathrm{SM}}+\frac{3-2 r_{m}-2 s_{W}^{2}}{4\left(1-r_{m}\right)^{2}} g_{Z} s_{W}^{2} \epsilon^{2}$ & $g_{e_{L}}^{\mathrm{SM}} \cos \theta$ \\
\hline$g_{e_{R}}$ & $g_{Z} s_{W}^{2}$ & $g_{e_{R}}^{\mathrm{SM}}$ & $g_{e_{R}}^{\mathrm{SM}}+\frac{2-2 r_{m}-s_{W}^{2}}{2\left(1-r_{m}\right)^{2}} g_{Z} s_{W}^{2} \epsilon^{2}$ & $g_{e_{R}}^{\mathrm{SM}} \cos \theta$ \\
\hline$g_{u_{L}}$ & $g_{Z}\left(\frac{1}{2}-\frac{2}{3} s_{W}^{2}\right)$ & $g_{u_{L}}^{\mathrm{SM}}$ & $g_{u_{L}}^{\mathrm{SM}}-\frac{5-2 r_{m}-4 s_{W}^{2}}{12\left(1-r_{m}\right)^{2}} g_{Z} s_{W}^{2} \epsilon^{2}$ & $g_{u_{L}}^{\mathrm{SM}} \cos \theta$ \\
\hline$g_{u_{R}}$ & $g_{Z}\left(-\frac{2}{3} s_{W}^{2}\right)$ & $g_{u_{R}}^{\mathrm{SM}}$ & $g_{u_{R}}^{\mathrm{SM}}-\frac{2-2 r_{m}-s_{W}^{2}}{3\left(1-r_{m}\right)^{2}} g_{Z} s_{W}^{2} \epsilon^{2}$ & $g_{u_{R}}^{\mathrm{SM}} \cos \theta$ \\
\hline$g_{d_{L}}$ & $g_{Z}\left(-\frac{1}{2}+\frac{1}{3} s_{W}^{2}\right)$ & $g_{d_{L}}^{\mathrm{SM}}$ & $g_{d_{L}}^{\mathrm{SM}}+\frac{1+2 r_{m}-2 s_{W}^{2}}{12\left(1-r_{m}\right)^{2}} g_{Z} s_{W}^{2} \epsilon^{2}$ & $g_{d_{L}}^{\mathrm{SM}} \cos \theta$ \\
\hline$g_{d_{R}}$ & $g_{Z}\left(\frac{1}{3} s_{W}^{2}\right)$ & $g_{d_{R}}^{\mathrm{SM}}$ & $g_{d_{R}}^{\mathrm{SM}}+\frac{2-2 r_{m}-s_{W}^{2}}{6\left(1-r_{m}\right)^{2}} g_{Z} s_{W}^{2} \epsilon^{2}$ & $g_{d_{R}}^{\mathrm{SM}} \cos \theta$ \\
\hline$g_{e_{L}}^{\prime}$ & 0 & $Q_{e_{L}}^{\prime} g^{\prime}$ & $\frac{c_{W}^{2}-r_{m} / 2}{1-r_{m}} \epsilon g_{Z} s_{W}$ & $g_{e_{L}}^{\mathrm{SM}} \sin \theta$ \\
\hline$g_{e_{R}}^{\prime}$ & 0 & $Q_{e_{R}}^{\prime} g^{\prime}$ & $\frac{c_{W}^{2}-r_{m}}{1-r_{m}} \epsilon g_{Z} s_{W}$ & $g_{e_{R}}^{\mathrm{SM}} \sin \theta$ \\
\hline$g_{u_{L}}^{\prime}$ & 0 & $Q_{u_{L}}^{\prime} g^{\prime}$ & $\frac{r_{m}-4 c_{W}^{2}}{6\left(1-r_{m}\right)} \epsilon g_{Z} s_{W}$ & $g_{u_{L}}^{\mathrm{SM}} \sin \theta$ \\
\hline$g_{u_{R}}^{\prime}$ & 0 & $Q_{u_{R}}^{\prime} g^{\prime}$ & $\frac{2\left(r_{m}-c_{W}^{2}\right)}{3\left(1-r_{m}\right)} \epsilon g_{Z} s_{W}$ & $g_{u_{R}}^{\mathrm{SM}} \sin \theta$ \\
\hline$g_{d_{L}}^{\prime}$ & 0 & $Q_{d_{L}}^{\prime} g^{\prime}$ & $\frac{r_{m}+2 c_{W}^{2}}{6\left(1-r_{m}\right)} \epsilon g_{Z} s_{W}$ & $g_{d_{L}}^{\mathrm{SM}} \sin \theta$ \\
\hline$g_{d_{R}}^{\prime}$ & 0 & $Q_{d_{R}}^{\prime} g^{\prime}$ & $\frac{c_{W}^{2}-r_{m}}{3\left(1-r_{m}\right)} \epsilon g_{Z} s_{W}$ & $g_{d_{R}}^{\mathrm{SM}} \sin \theta$ \\
\hline$A^{\mathrm{PV}}(p)$ & eq. $(2.10)$ & eq. $(3.3)$ & eq. $(3.14)$ & eq. $(3.13)$ \\
\hline$A^{\mathrm{PV}}\left({ }^{12} \mathrm{C}\right)$ & eq. (2.11) & eq. (3.3) & eq. (3.15) & eq. (3.13) \\
\hline
\end{tabular}

Table 1. Analytical expressions for the effective couplings of $Z$ and $Z^{\prime}$ bosons to the SM quarks and charged leptons in the $\mathrm{SM}$, chiral $\mathrm{U}(1)^{\prime}$ models (from $Q_{f_{L}} \neq Q_{f_{R}}$ ) and non-chiral U(1)' models (from the kinetic mixing $\epsilon B^{\mu \nu} F_{\mu \nu}^{\prime}$ and mass mixing $\delta m^{2} Z^{\mu} Z_{\mu}^{\prime}$ ). The last two rows are the corresponding analytic formulae of $A^{\mathrm{PV}}$ for electron scattering off proton or ${ }^{12} \mathrm{C}$. The superscript "SM" indicates SM contributions. Here $g_{Z} \equiv e / s_{W} c_{W}, s_{W} \equiv \sin \theta_{W}$ being the weak mixing angle (and $\left.c_{W} \equiv \cos \theta_{W}\right)$ and we have defined $r_{m} \equiv m_{Z^{\prime}}^{2} / m_{Z}^{2}$. See the main text for details.

where $\overline{u_{3}}\left(u_{1}\right)$ and $\overline{u_{4}}\left(u_{2}\right)$ denote the final (initial) electron and nucleon states, and

$$
G_{L, R}=\frac{-e^{2}}{q^{2}}+\frac{g_{e_{L}, e_{R}} g_{V}}{q^{2}-m_{Z}^{2}}+\frac{g_{e_{L}, e_{R}}^{\prime} g_{V}^{\prime}}{q^{2}-m_{Z^{\prime}}^{2}}
$$

contains contributions from the $t$-channel $\gamma, Z$, and $Z^{\prime}$ diagrams, respectively. Applying the standard trace technology, it is straightforward to obtain

$$
\frac{\left|\mathcal{M}_{L}\right|^{2}}{\left|\mathcal{M}_{R}\right|^{2}}=\frac{G_{L}^{2}}{G_{R}^{2}}
$$


which, according to eq. (1.1), implies

$$
A^{\mathrm{PV}}=\frac{G_{R}^{2}-G_{L}^{2}}{G_{R}^{2}+G_{L}^{2}}
$$

Now substituting eq. (2.5) in eq. (2.7), we obtain

$$
A^{\mathrm{PV}} \approx \frac{\left(g_{e_{L}}-g_{e_{R}}\right) g_{V}}{4 \pi \alpha} \frac{Q^{2}}{m_{Z}^{2}}+\frac{\left(g_{e_{L}}^{\prime}-g_{e_{R}}^{\prime}\right) g_{V}^{\prime}}{4 \pi \alpha} \frac{1}{1+m_{Z^{\prime}}^{2} / Q^{2}},
$$

where $Q^{2} \equiv-q^{2}>0$ and $\alpha \equiv e^{2} / 4 \pi$ is the fine-structure constant. In deriving eq. (2.8), we have made the following approximations:

$$
\frac{Q^{2}}{m_{Z}^{2}} \ll 1, \quad \text { and } \quad \frac{g_{e}^{\prime} g_{V}^{\prime}}{1+m_{Z^{\prime}}^{2} / Q^{2}} \ll 1
$$

where $g_{e}^{\prime} \sim \max \left(g_{e_{L}}^{\prime}, g_{e_{R}}^{\prime}\right)$. The exact expression of $A^{\mathrm{PV}}$ is lengthy and not very helpful. Within a theory containing an additional $Z^{\prime}$ boson, once the six effective couplings $\left(g_{e_{L}}, g_{e_{R}}, g_{V}, g_{e_{L}}^{\prime}, g_{e_{R}}^{\prime}, g_{V}^{\prime}\right)$ are known, $A^{\mathrm{PV}}$ can be obtained using eq. (2.8) and confronted with its existing or future constraints, as exemplified in the following two sections.

We stress that eq. (2.8) is obtained from the most general Lagrangian in eq. (2.1). In the limit of $g_{e_{L}}^{\prime}=g_{e_{R}}^{\prime}, g_{V}^{\prime} \rightarrow 0$ or $m_{Z^{\prime}} \rightarrow \infty$, the BSM contributions are vanishing, and taking the SM values in table 1 and using eq. (2.2), we can easily reproduce the leading-order $\mathrm{SM}$ expressions of the parity-violating asymmetries $A^{\mathrm{PV}}[13]$ :

$$
\begin{aligned}
A_{\mathrm{SM}}^{\mathrm{PV}}(e+p) & \approx-\frac{G_{F} Q^{2}\left(1-4 s_{W}^{2}\right)}{4 \sqrt{2} \pi \alpha}, \\
A_{\mathrm{SM}}^{\mathrm{PV}}\left(e+{ }^{12} \mathrm{C}\right) & \approx \frac{3 \sqrt{2} G_{F} Q^{2} s_{W}^{2}}{\pi \alpha},
\end{aligned}
$$

for electron-proton scattering $(\mathcal{N}=0$ and $\mathcal{Z}=1)$ and electron- ${ }^{12} \mathrm{C}$ scattering $(\mathcal{N}=\mathcal{Z}=6)$, respectively. Here $G_{F}=\pi \alpha /\left(\sqrt{2} m_{W}^{2} s_{W}^{2}\right)$ is the Fermi constant.

\section{New physics effects}

This section deals with obtaining the couplings of an additional $Z^{\prime}$ boson with the SM fermions in some representative $\mathrm{U}(1)^{\prime}$ models to study the P2 prospects. We first discuss three chiral $\mathrm{U}(1)^{\prime}$ models with explicit parity-violation, and then turn to parity-conserving $\mathrm{U}(1)^{\prime}$ models with mass or kinetic $Z-Z^{\prime}$ mixing.

\subsection{Chiral $\mathrm{U}(1)^{\prime}$ models}

First let us consider new $\mathrm{U}(1)^{\prime}$ gauge symmetries under which $e_{L}$ and $e_{R}$ have different charges. We refer to such models as chiral $\mathrm{U}(1)^{\prime}$ models. Anomaly cancellation usually requires the existence of three right-handed neutrinos $\nu_{R}$ (for simplicity we have neglected 


\begin{tabular}{|cc|cccc|}
\hline \multirow{2}{*}{ model } & \multirow{2}{*}{$(x, y)$} & \multicolumn{4}{|c|}{$\mathrm{U}(1)^{\prime}$ charge assignment } \\
\cline { 3 - 6 } & & $\left(\nu_{L}, e_{L}\right)$ & $\left(\nu_{R}, e_{R}\right)$ & $\left(u_{L}, d_{L}\right)$ & $\left(u_{R}, d_{R}\right)$ \\
\cline { 3 - 6 } & $-\frac{3}{2}(x+y)$ & $-(x+2 y, 2 x+y)$ & $\frac{1}{2}(x+y)$ & $(x, y)$ \\
\hline $\mathrm{U}(1)_{L}^{\prime}$ & $\left(-\frac{2}{3}, \frac{4}{3}\right)$ & -1 & $(-2,0)$ & $\frac{1}{3}$ & $\left(-\frac{2}{3}, \frac{4}{3}\right)$ \\
$\mathrm{U}(1)_{R}^{\prime}$ & $(1,-1)$ & 0 & $(1,-1)$ & 0 & $(1,-1)$ \\
$\mathrm{U}(1)_{X}^{\prime}$ & $(0,1)$ & $-\frac{3}{2}$ & $(-2,-1)$ & $\frac{1}{2}$ & $(0,1)$ \\
\hline
\end{tabular}

Table 2. Quantum numbers of fermions in the three chiral $\mathrm{U}(1)^{\prime}$ examples considered here.

the generation indices $i=1,2,3)$, and implies the following charge assignments of chiral fermions $[49,50]$ :

$$
\begin{array}{lll}
Q_{u_{R}}^{\prime}=x, & Q_{d_{R}}^{\prime}=y, & Q_{\nu_{R}}^{\prime}=-x-2 y, \\
Q_{e_{R}}^{\prime}=-2 x-y, & Q_{Q_{L}}^{\prime}=\frac{1}{2}(x+y), & Q_{L_{L}}^{\prime}=-\frac{3}{2}(x+y) .
\end{array}
$$

Here $Q_{L}=\left(u_{L}, d_{L}\right)^{\top}$ and $L_{L}=\left(\nu_{L}, e_{L}\right)^{\top}$ are respectively the left-handed quark and lepton doublets, and $Q_{f}^{\prime}$ is the $\mathrm{U}(1)^{\prime}$ charge of the chiral fermion $f$. The charges $x$ and $y$ can be arbitrary integers or fractional numbers. However, from the model building perspective, simple integers or fractions are preferred in order to construct simple (low-dimensional) gauge-invariant operators. For simplicity, we will assume that the right-handed neutrinos are heavier than $m_{Z^{\prime}} / 2$, such that the decay channel $Z^{\prime} \rightarrow \nu_{R} \bar{\nu}_{R}$ is kinematically forbidden and the $\nu_{R}$ do not have any effect on the P2 sensitivities.

The simplest example is perhaps the $\mathrm{U}(1)_{R}^{\prime}$ model given in table 2 . In this model, all right-handed fermions are charged under $\mathrm{U}(1)_{R}^{\prime}$, and none of the left-handed fermions are, i.e. $(x, y)=(1,-1)$ in eq. $(3.1)$; see e.g. refs. [51-53]. One might also consider the opposite case where all left-handed fermions are charged and none of the right-handed fermions are. Unfortunately for such assignment it is impossible to achieve chiral anomaly cancellation. If, for instance, $e_{R}$ is not charged under the chiral $\mathrm{U}(1)^{\prime}$, which corresponds to $x / y=-1 / 2$ in eq. (3.1), then the only solution is where the other right-handed fermions are charged for anomaly cancellation. This is the $\mathrm{U}(1)_{L}^{\prime}$ case shown in table 2 . In addition to $\mathrm{U}(1)_{R}^{\prime}$ and $\mathrm{U}(1)_{L}^{\prime}$, we choose another solution with $(x, y)=(0,1)$, where right-handed up-quarks do not couple of $Z^{\prime}$, and refer to it as $\mathrm{U}(1)_{X}^{\prime}$ in table 2 . Obviously there are many more possibilities. Our choice of the three models summarized in table 2 is motivated by the fact that they are typical for different corners of the parameter space to be explored at P2. For instance, the $\mathrm{U}(1)_{R}$ model will not contribute to the asymmetry in electron- ${ }^{12} \mathrm{C}$ scattering (cf. eq. (3.5) below).

Computing $A^{\mathrm{PV}}$ for chiral $\mathrm{U}(1)^{\prime}$ models is now straightforward. In the absence of $Z-Z^{\prime}$ mixing, the $Z$ couplings are not modified and the $Z^{\prime}$ couplings in eq. (2.1) are given by

$$
g_{f}^{\prime}=g^{\prime} Q_{f}^{\prime},
$$


where $g^{\prime}$ is the fundamental gauge coupling of $\mathrm{U}(1)^{\prime}$. Using eq. (2.8), we obtain

$$
\Delta A^{\mathrm{PV}} \equiv A^{\mathrm{PV}}-A_{\mathrm{SM}}^{\mathrm{PV}} \approx \frac{g^{\prime 2} Q_{N}^{\prime} Q_{L R}^{\prime}}{4 \pi \alpha} \frac{1}{1+m_{Z^{\prime}}^{2} / Q^{2}},
$$

where $Q_{L R}^{\prime} \equiv Q_{e_{L}}^{\prime}-Q_{e_{R}}^{\prime}$, and $Q_{N}^{\prime}$ is either the weak charge $Q_{p}^{\prime}$ of the proton or the weak charge $Q_{12}^{\prime}$ of ${ }^{12} \mathrm{C}$, with

$$
\begin{aligned}
Q_{p}^{\prime} & \equiv Q_{u_{L}}^{\prime}+Q_{u_{R}}^{\prime}+\frac{1}{2}\left(Q_{d_{L}}^{\prime}+Q_{d_{R}}^{\prime}\right), \\
Q_{{ }_{12} \mathrm{C}}^{\prime} & \equiv 9\left(Q_{u_{L}}^{\prime}+Q_{u_{R}}^{\prime}+Q_{d_{L}}^{\prime}+Q_{d_{R}}^{\prime}\right) .
\end{aligned}
$$

From table 2 , we note that in the $\mathrm{U}(1)_{R}^{\prime}$ model, $Q_{{ }_{12} \mathrm{C}}^{\prime}$ vanishes, which implies that electron${ }^{12} \mathrm{C}$ scattering has no sensitivity to $Z^{\prime}$ in this model.

Here we comment on the potential effect of incoherence in $e+{ }^{12} \mathrm{C}$ scattering and the validity of summing up the charges in eq. (3.5). In the $\mathrm{P} 2$ experiment, $Q \approx 93 \mathrm{MeV}$ is comparable to the inverse of the ${ }^{12} \mathrm{C}$ nucleus radius and there is a considerable amount of loss of coherence, which is usually taken into account by including a form factor depending on the nucleon distributions. Due to the small difference between neutron and proton distributions, two types of form factors are often considered, namely weak and charge form factors, denoted respectively by $F_{W}\left(Q^{2}\right)$ and $F_{\mathrm{Ch}}\left(Q^{2}\right)$. The form factor $F_{\mathrm{Ch}}\left(Q^{2}\right)$ depends on the proton distribution in the nucleus, while $F_{W}\left(Q^{2}\right)$ is determined mainly by the neutron distribution, as the coupling of $Z$ to neutrons is significantly larger than the coupling of $Z$ to protons. In presence of the couplings of $Z^{\prime}$ to protons and neutrons, the cross sections $\sigma_{L, R}$ depend on the combination of $F_{W}\left(Q^{2}\right)-F_{\mathrm{Ch}}\left(Q^{2}\right)$. Using the Helm analytic approximation for form factors [54], we find that the two form factors $F_{W, \mathrm{Ch}}\left(Q^{2}\right)$ are both around 0.75 with percent-level uncertainties for ${ }^{12} \mathrm{C}$ at $Q \approx 93 \mathrm{MeV}$. This implies that with the approximation of $F_{W}\left(Q^{2}\right) \simeq F_{\mathrm{Ch}}\left(Q^{2}\right)$ the effect of incoherence cancels out for $A^{\mathrm{PV}}$, which justifies eq. (3.5). It should be noted that this justification does not depend on the details of $\mathrm{U}(1)^{\prime}$ models and applies also to all the non-chiral $\mathrm{U}(1)^{\prime}$ models discussed below.

\subsection{Non-chiral U(1)' models and mixing-induced couplings}

There are plenty of non-chiral $\mathrm{U}(1)^{\prime}$ models with parity-conserving charge assignments. For instance, the $\mathrm{U}(1)_{B-L}$ model [55-57], which assigns all leptons (both left- and righthanded) a charge of -1 and all quarks a charge of $\frac{1}{3}$, is among the most extensively studied models in the literature. Although the non-chiral charge assignments do not cause any additional source of asymmetry in polarized electron scattering, constraints on non-chiral $\mathrm{U}(1)^{\prime}$ models are possible in the presence of $Z-Z^{\prime}$ mixing, as illustrated by the lower right diagram in figure 1.

Let us consider that the $Z-Z^{\prime}$ mixing arises from the following terms:

$$
\mathcal{L} \supset-\frac{\epsilon}{2} B^{\mu \nu} F_{\mu \nu}^{\prime}+\delta m^{2} \hat{Z}^{\mu} \hat{Z}_{\mu}^{\prime}
$$

where $B^{\mu \nu}$ and $F_{\mu \nu}^{\prime}$ are the field strength tensors of hypercharge $\mathrm{U}(1)_{Y}$ and $\mathrm{U}(1)^{\prime}$, respectively. The second term is a mass-mixing term which exists if the symmetry breaking of 
$\mathrm{U}(1)^{\prime}$ is not decoupled from the electroweak symmetry breaking. We define a mass mixing parameter $\theta$ by

$$
\tan \theta \equiv \frac{\delta m^{2}}{m_{Z^{\prime}}^{2}-m_{Z}^{2}}
$$

where $m_{Z}^{2}$ is the $Z$ boson mass. Note that in the presence of eq. (3.6), the kinetic terms are not canonical and the mass matrix is not diagonal. One needs to perform necessary transformations and redefine $Z$ and $Z^{\prime}$ as the physical mass eigenstates. To avoid potential confusion, we have denoted the original states as $\hat{Z}$ and $\hat{Z}^{\prime}$ in eq. (3.6). Following the notation in ref. [58], the couplings in the physical basis can be written as

$$
\mathcal{L} \supset Z_{\mu}\left[J_{\mathrm{NC}}^{\mu} \cos \beta-J_{X}^{\mu} \sin \beta\right]+Z_{\mu}^{\prime}\left[J_{\mathrm{NC}}^{\mu} \sin \beta+J_{X}^{\mu} \cos \beta\right],
$$

where the effective $Z-Z^{\prime}$ mixing angle is

$$
\tan \beta \approx \tan \theta+\frac{\epsilon s_{W}}{r_{m}-1}+\mathcal{O}\left(\theta^{2}, \epsilon^{2}\right)
$$

where we have defined the mass ratio

$$
r_{m} \equiv \frac{m_{Z^{\prime}}^{2}}{m_{Z}^{2}}
$$

Eq. (3.9) incorporates contributions from the mass mixing $\theta$ and the kinetic mixing $\epsilon$. The $\mathrm{SM} \mathrm{NC} J_{\mathrm{NC}}^{\mu}$ and the $Z^{\prime}$-induced neutral current $J_{X}^{\mu}$ in eq. (3.9) are defined respectively as

$$
\begin{aligned}
J_{\mathrm{NC}}^{\mu} & =\sum_{f} g_{f}^{\mathrm{SM}} \bar{f} \gamma^{\mu} f \\
J_{X}^{\mu} & =\sum_{f} \frac{\bar{f} \gamma^{\mu} f}{\sqrt{1-\epsilon^{2}}}\left[g^{\prime} Q_{f}^{\prime}-g_{Z} \epsilon s_{W} Y_{f}\right],
\end{aligned}
$$

with $g_{f}^{\mathrm{SM}}$ the SM gauge couplings for the chiral fermions $f$ collected in the second column of table 1. In eq. (3.12), $g_{Z} \equiv g / c_{W}=\sqrt{4 \pi \alpha} / s_{W} c_{W}$ is a SM coupling, and $Y_{f}$ is the SM $\mathrm{U}(1)_{Y}$ charge for the fermions.

From eqs. (3.8)-(3.12) it is straightforward to derive the effective couplings of $Z$ and $Z^{\prime}$ to fermions; these are summarized in table 1 . Note that for $Z$ couplings we have expanded the results to order $\epsilon^{2}$ because the $Z$ diagram with one vertex modified by $\epsilon^{2}$ and the $Z^{\prime}$ diagram with two vertices proportional to $\epsilon$ have comparable contributions to the parity-violating asymmetry.

Now using the effective couplings and eq. (2.8), we can compute the mixing-induced contribution to the parity-violating asymmetry, $\Delta A^{\mathrm{PV}} \equiv A^{\mathrm{PV}}-A_{\mathrm{SM}}^{\mathrm{PV}}$, for non-chiral $\mathrm{U}(1)^{\prime}$ models. The result, in the presence of only mass-mixing, is

$$
\frac{\Delta A^{\mathrm{PV}}}{A_{\mathrm{SM}}^{\mathrm{PV}}}=\sin ^{2} \theta\left(\frac{m_{Z}^{2}}{m_{Z^{\prime}}^{2}+Q^{2}}-1\right) .
$$

For only kinetic mixing, the result is

$$
\begin{aligned}
\frac{\Delta A^{\mathrm{PV}}}{A_{\mathrm{SM}}^{\mathrm{PV}}}(e+p) & =\frac{m_{Z^{\prime}}^{2}\left(1-r_{m}\right)\left(1-4 s_{W}^{2}\right)+Q^{2}\left(4 r_{m} s_{W}^{2}+2 r_{m}-3\right)}{\left(1-r_{m}\right)^{2}\left(1-4 s_{W}^{2}\right)\left(m_{Z^{\prime}}^{2}+Q^{2}\right)} s_{W}^{2} \epsilon^{2}, \\
\frac{\Delta A^{\mathrm{PV}}}{A_{\mathrm{SM}}^{\mathrm{PV}}}\left(e+{ }^{12} \mathrm{C}\right) & =\frac{m_{Z^{\prime}}^{2} s_{W}^{2}\left(1-r_{m}\right)+Q^{2}\left(1-r_{m}-r_{m} s_{W}^{2}\right)}{\left(1-r_{m}\right)^{2}\left(m_{Z^{\prime}}^{2}+Q^{2}\right)} \epsilon^{2},
\end{aligned}
$$

for electron-proton and electron- ${ }^{12} \mathrm{C}$ scattering, respectively. 
When both mass and kinetic mixing are present, one can add their contributions linearly as long as both $\epsilon$ and $\theta$ are perturbatively small. In the above results, we have neglected the contribution of the fundamental gauge coupling $g^{\prime}$ in eq. (3.12). Although for non-chiral $\mathrm{U}(1)^{\prime}$ models this part does not account for parity violation, sizable values of $g^{\prime}$ do affect the P2 sensitivity by changing the effective couplings to nucleons if $g^{\prime}$ is comparable to $\theta$ or $\epsilon$. This effect can be readily included by adding a correction of $g^{\prime} Q_{f}^{\prime}$ to the mixing-induced couplings as long as both are perturbatively small. In this case, the P2 sensitivity to $\epsilon$ and $\theta$ becomes model-dependent. We select two non-chiral $\mathrm{U}(1)^{\prime}$ models when the model-dependent details are required, $\mathrm{U}(1)_{B-L}$ as aforementioned, and $\mathrm{U}(1)_{B}$ which gauges the baryon number only [59]. For these models with sizable $g^{\prime}$ and $\theta$, the new physics contributions can be formulated as

$$
\frac{\Delta A^{\mathrm{PV}}}{A_{\mathrm{SM}}^{\mathrm{PV}}}=\sin ^{2} \theta\left[\frac{m_{Z}^{2}(1+R)}{m_{Z^{\prime}}^{2}+Q^{2}}-1\right]
$$

where $R$ takes the following expressions for the $\mathrm{U}(1)_{B}$ model:

$$
\begin{aligned}
R_{e+p}\left[\mathrm{U}(1)_{B}\right] & =\frac{12 g^{\prime}}{g_{Z} \sin \theta\left(1-4 s_{W}^{2}\right)}, \\
R_{e+{ }^{12} \mathrm{C}}\left[\mathrm{U}(1)_{B}\right] & =-\frac{6 g^{\prime}}{g_{Z} \sin \theta s_{W}^{2}} .
\end{aligned}
$$

For $\mathrm{U}(1)_{B-L}$, we simply need to multiply the $R$ factors in eqs. (3.17) and (3.18) by a factor of $-1 / 3$, i.e.

$$
R\left[\mathrm{U}(1)_{B-L}\right]=-\frac{1}{3} R\left[\mathrm{U}(1)_{B}\right]
$$

\section{$4 \quad$ P2 sensitivity study}

In this section, we derive the P2 sensitivity for the U(1) models discussed in the previous section.

\subsection{Method}

The P2 experiment will measure polarized electron scattering with an electron beam of $155 \mathrm{MeV}$ [60]. The momentum transfer $Q^{2}$ in this process can be determined by

$$
Q^{2} \approx 4 E_{i} E_{f} \sin \left(\theta_{f} / 2\right)
$$

where $\theta_{f}$ is the scattering angle of the outgoing electron with respect to the incoming one, and $E_{f} \approx E_{i} \approx 155 \mathrm{MeV}$ are the final/initial electron energies. The angular acceptance of the detector is

$$
\theta_{f} \in\left[\bar{\theta}_{f}-\frac{1}{2} \delta \theta_{f}, \bar{\theta}_{f}+\frac{1}{2} \delta \theta_{f}\right], \quad \text { with } \quad \bar{\theta}_{f}=35^{\circ}, \delta \theta_{f}=20^{\circ} .
$$

In our analysis we take $Q^{2} \approx(93 \mathrm{MeV})^{2}$ evaluated from the central value $\bar{\theta}_{f}$. For $e+p$ scattering, the expected value of $A^{\mathrm{PV}}$ is $39.94 \times 10^{-9}$ and the $\mathrm{P} 2$ experiment will be able 
to measure it with an uncertainty of $\Delta A^{\mathrm{PV}}=0.56 \times 10^{-9}$, which corresponds to a relative uncertainty of

$$
\frac{\Delta A^{\mathrm{PV}}}{A^{\mathrm{PV}}} \approx 1.4 \%, \quad(e+p \text { scattering }) .
$$

This would imply a relative uncertainty of $0.14 \%$ for $s_{W}^{2}$. For $e+{ }^{12} \mathrm{C}$ scattering, the achievable uncertainty is

$$
\frac{\Delta A^{\mathrm{PV}}}{A^{\mathrm{PV}}} \approx 0.3 \%, \quad\left(e+{ }^{12} \mathrm{C} \text { scattering }\right)
$$

Since $A^{\mathrm{PV}}\left({ }^{12} \mathrm{C}\right)$ is proportional to $s_{W}^{2}$ rather than $1-4 s_{W}^{2}$, the relative uncertainty for $s_{W}^{2}$ is also $0.3 \%$.

With the experimental setup described above and the new physics contributions formulated in eqs. (3.3) and (3.13) to (3.15), it is straightforward to study the sensitivity of $\mathrm{P} 2$ to $Z^{\prime}$ models. By requiring that the new physics contributions do not exceed

$$
\frac{\Delta A^{\mathrm{PV}}}{A^{\mathrm{PV}}}= \begin{cases}\sqrt{2.71} \times 1.4 \%=2.30 \% & (\text { for } e+p \text { scattering }), \\ \sqrt{2.71} \times 0.3 \%=0.49 \% & \left(\text { for } e+{ }^{12} \mathrm{C} \text { scattering }\right)\end{cases}
$$

where the factor $\sqrt{2.71}$ converts $1 \sigma$ to $90 \%$ confidence level (C.L.), we obtain the P2 sensitivity curves in figures 2 to 6 for the variety of $\mathrm{U}(1)^{\prime}$ models under study.

As for the value of the weak mixing angle $\theta_{W}$ used in our numerical calculations, it is precisely measured at the electroweak scale from $Z$ pole observables, the $W$ boson mass and a variety of $\mathrm{NC}$ processes. In the global fitting of high-energy data, it is determined to be $s_{W}^{2}=0.23122 \pm 0.00003$ [1], which can be run down via the SM renormalization group equations to lower energies $[61,62]$ and compared to the direct measurements of the weak mixing angle at that low-energy scales [63]. In the numerical calculations, we have fixed the value of $s_{W}^{2}$ to its expected central low- $q^{2}$ value of $0.230 \pm 0.00003$. As shown in eq. (2.10), in the SM the parity-violating asymmetry $A_{\mathrm{SM}}^{\mathrm{PV}}$ in electron-proton scattering is proportional to the factor of $\left(1-4 s_{W}^{2}\right)$, therefore the uncertainty of $A_{\mathrm{SM}}^{\mathrm{PV}}$ due to the error bars $\Delta s_{W}^{2}$ of $s_{W}^{2}$ goes like

$$
\Delta A_{\mathrm{SM}}^{\mathrm{PV}}(e+p) \propto \frac{-4 \Delta s_{W}^{2}}{1-4 s_{W}^{2}} .
$$

For scattering with ${ }^{12} \mathrm{C}$, the $\mathrm{SM} A^{\mathrm{PV}}$ depends linearly on the weak mixing angle, i.e.

$$
\Delta A_{\mathrm{SM}}^{\mathrm{PV}}\left(e+{ }^{12} \mathrm{C}\right) \propto \frac{\Delta s_{W}^{2}}{s_{W}^{2}} .
$$

In the case of $Z-Z^{\prime}$ mixing, the new physics contributions to $A^{\mathrm{PV}}$ are also subject to the weak mixing angle uncertainties, as implied in eqs. (3.9) to (3.12). They enter the result in the form of $\epsilon \Delta s_{W}^{2}$ or $\sin \theta \Delta s_{W}^{2}$. In light of the small experimental uncertainties of $s_{W}^{2}$, we neglect this higher-order effect in this paper.

Since the measurable $A^{\mathrm{PV}}$ is a single number, there is an unavoidable degeneracy between new physics and a variation of $s_{W}^{2}$. This could, however, be resolved by performing the P2 measurements not only of the total asymmetry but of the angular distribution of the 
cross-sections over the whole range of scattering angle $\theta_{f}$ (see eq. (4.2)). In this way one could perform measurements at different $Q^{2}$, see eq. (4.1), which could for appropriate $Z^{\prime}$ masses break the degeneracy of the Weinberg angle with new physics contributions to the asymmetry. Of course, measuring at two $Q^{2}$ with the same amount of available beam-time will cost precision. In case interesting departures from the expectation are found during the run-time of the experiment, a trade-off between precision and new physics sensitivity could be a possibility. We note that a measurement at large scattering angle is foreseen by $\mathrm{P} 2$, in order to measure corrections from the proton structure to the measurement [13].

\subsection{Current experimental limits}

Before discussing our sensitivity results for P2, we briefly review the existing bounds on $Z^{\prime}$ in each of the $\mathrm{U}(1)^{\prime}$ models considered in section 3 , following the compilations in, e.g. refs. [64-69]. Since the P2 experiment is based on electron-nucleon scattering, bounds on electron and quark couplings should be taken into account. In addition, for specific models, bounds on neutrino or muon couplings can also be used to constrain $g^{\prime}, \epsilon$, and $\theta$. In addition, gauge invariance links electron scattering to neutrino scattering.

Atomic parity violation. Precise low-energy measurements of APV have been performed using atomic ${ }^{133} \mathrm{Cs}[24-27],{ }^{205} \mathrm{Tl}[28,29],{ }^{208} \mathrm{~Pb}[30],{ }^{209} \mathrm{Bi}[31]$ and $100,102,104,106 \mathrm{Yb}[32]$, and the most precise ones are the $6 S_{1 / 2}-7 S_{1 / 2}$ nuclear transition in ${ }^{133} \mathrm{Cs}$. To lowest order, the nuclear weak charge measured in APV is given by $Q_{W}=-\mathcal{N}_{\mathrm{Cs}}+\mathcal{Z}_{\mathrm{Cs}}\left(1-4 s_{W}^{2}\right)$, with $\mathcal{Z}_{\mathrm{Cs}}=55$ and $\mathcal{N}_{\mathrm{Cs}}=78$ being the proton and neutron numbers in ${ }^{133} \mathrm{Cs}$ respectively. Combining calculation of the nuclear spin-independent parity-violating electric dipole transition amplitude and the most accurate experimental measurements [33, 48, 70, 71], we have the following SM predictions and experimental values of $Q_{W}\left({ }^{133} \mathrm{Cs}\right)$ :

$$
Q_{W}^{\mathrm{SM}}\left({ }^{133} \mathrm{Cs}\right)=-73.23(1), \quad Q_{W}^{\exp }\left({ }^{133} \mathrm{Cs}\right)=-73.71(35),
$$

which corresponds to an accuracy of $0.47 \%(0.77 \%)$ at the $1 \sigma(90 \%)$ C.L., and can be used to set limits on any new physics contributions to APV $[2,40,72,73]$. In presence of the $Z^{\prime}$ boson, the $6 S_{1 / 2}-7 S_{1 / 2}$ nuclear transition can be viewed as an electron-nucleus scattering in the Cesium atom, and the calculation methods for proton and ${ }^{12} \mathrm{C}$ can be applied directly to the case of ${ }^{133} \mathrm{Cs}$, for instance eqs. (3.3), (3.13) and (3.16). For concreteness, we set the energy scale of ${ }^{133} \mathrm{Cs}$ experiments to be $Q=30 \mathrm{MeV}$ [73], and the resultant limits are shown in figures 2 to 6 as the pink curves.

Beam dumps. Beam dump experiments such as E137 [74], E141 [75], E774 [76] and Orsay [77] have searched for particles that could be produced in electron scattering off nuclei in fixed targets and their subsequent decay to visible final states. In these experiments, the production process is electron bremsstrahlung and the detection relies on $Z^{\prime} \rightarrow e^{+} e^{-}$ decay. Therefore the searches are sensitive to the electron coupling of a low-mass $Z^{\prime}$ in the range of 1 to $100 \mathrm{MeV}$. We use the package DARKCAST [65] to recast and combine the bounds from these experiments. 
There was recently a search of $e N \rightarrow e N X$ at NA64, with the $X$ boson decaying invisibly [78]. The null result at this experiment (which is not yet incorporated in DARKCAST) sets an upper bound on the effective coupling of $X$ to electron, which can go up to the order of $10^{-5}$ for a $\mathrm{MeV}$-scale $X$ boson mass and up to $10^{-2}$ if $X$ is at the $\mathrm{GeV}$-scale, depending on the Lorentz structure of the couplings. When the NA64 limits in ref. [78] are cast onto the chiral and non-chiral $\mathrm{U}(1)^{\prime}$ models in this paper, the corresponding limits are expected to be the same order as that of the electron $g-2$ limits or up to one order of magnitude better (cf. figure 4 in ref. [78]), and are thus not shown in figures 2 to 6 .

Collider searches. New $Z^{\prime}$ gauge bosons could be probed at colliders in several different ways. For low-mass $Z^{\prime}$ at $e^{+} e^{-}$colliders, the mono- $\gamma$ channel $e^{+} e^{-} \rightarrow \gamma Z^{\prime}$, followed by decays $Z^{\prime} \rightarrow e^{+} e^{-}$or $\mu^{+} \mu^{-}$or into invisible states, is usually considered as the most restrictive one. Searches in these channels have been performed at BaBar [79, 80], NA48/2 [81], KLOE [82] and LEP [83]. At hadron colliders, the Drell-Yan process $p p \rightarrow Z^{\prime} \rightarrow \mu^{+} \mu^{-}$ or dijet, searched for by LHCb [84], ATLAS [85, 86], and CMS [87, 88], produces the leading constraints on heavy $Z^{\prime}$ above the $Z$ pole. Besides, the LEP data can also be used to constrain four-lepton effective operators generated by heavy $Z^{\prime}$ [89]. We adopt the ATLAS/CMS dijet limit from figure 3 in ref. [90]. Other collider bounds are obtained using the DARKCAST package [65]. The LEP bounds in figures 2 and 6 (absent in figures 3 to 5 due to insignificance) are derived by fitting four-lepton effective operators to precision measurements of $e^{+} e^{-} \rightarrow \ell^{+} \ell^{-}$at LEP [89]. ${ }^{1}$

Neutrino scattering. Elastic neutrino scattering data collected by CHARM II [92, 93], LSND [94], Borexino [95], TEXONO [96] and COHERENT [97] are sensitive to a low-mass $Z^{\prime}$ as it could mediate a $t$-channel process in $\nu+e^{-}$or $\nu+N$ scattering enhanced by the low mass and low momentum transfer. According to studies in refs. [58, 73, 98, 99], we select two of the most restrictive sets of data, CHARM II and TEXONO, and perform an independent data fitting ${ }^{2}$ for the various models considered in this work. The neutrino scattering bounds apply to most of the models except for $\mathrm{U}(1)_{R}$ and $\mathrm{U}(1)_{B}$, in which lefthanded neutrinos $\nu_{L}$ are not charged under the new $\mathrm{U}(1)$. However, in the presence of kinetic/mass mixing, these models could still be constrained by neutrino scattering.

Lepton anomalous magnetic moment. The muon and electron anomalous magnetic moments are sensitive to generic neutral bosons coupled to them. The $Z^{\prime}$ contribution to $a_{\ell} \equiv(g-2) / 2$ can be evaluated by [100]:

$$
\Delta a_{\ell}=\frac{1}{8 \pi^{2}}\left(g_{\ell}^{\prime} \epsilon_{Z^{\prime}}\right)^{2} \int_{0}^{1} \frac{2 x^{2}(1-x)}{(1-x)\left(1-x \epsilon_{Z^{\prime}}^{2}\right)+x \epsilon_{Z^{\prime}}^{2}} d x
$$

where $\epsilon_{Z^{\prime}} \equiv m_{\ell} / m_{Z^{\prime}}$ and $\ell=\mu$ or $e$. The muon $g-2$ has a long-standing $3.7 \sigma$ discrepancy between the experimental and the calculated SM values [101], i.e.

$$
\Delta a_{\mu} \equiv a_{\mu}^{\exp }-a_{\mu}^{\mathrm{SM}}=(27.9 \pm 7.6) \times 10^{-10} .
$$

\footnotetext{
${ }^{1}$ The LEP limits on flavored $Z^{\prime}$ bosons can be found e.g. in ref. [91].

${ }^{2}$ Code is available from https://github.com/xunjiexu/Dark_Z.
} 
The electron $g-2$ had earlier seen a $2.4 \sigma$ discrepancy in the opposite direction [102], based on the comparison between the SM prediction [103] and a measurement of the fine-structure constant using Cesium [104]:

$$
\Delta a_{e}^{\mathrm{Cs}} \equiv a_{e}^{\exp (\mathrm{Cs})}-a_{e}^{\mathrm{SM}}=(-8.7 \pm 3.6) \times 10^{-13} .
$$

However, a recent measurement of the fine-structure constant using Rubidium [105] has pushed the discrepancy to a mere $1.6 \sigma$ in the same direction as $(g-2)_{\mu}$ :

$$
\Delta a_{e}^{\mathrm{Rb}} \equiv a_{e}^{\exp (\mathrm{Rb})}-a_{e}^{\mathrm{SM}}=(4.8 \pm 3.0) \times 10^{-13} .
$$

Although this weakens the possibility of any new physics contribution to $\Delta a_{e}$, the Cs and $\mathrm{Rb}$ measurements of $\alpha$ now disagree by more than $5 \sigma$, which makes this whole issue quite murky. Here we take conservative bounds from $(g-2)_{\mu}$ and $(g-2)_{e}$ by requiring that $\Delta a_{\ell}$ does not exceed the experimental best-fit values given by eqs. (4.10) and (4.11) by $5 \sigma$. These bounds are presented in figures 2 to 6 as green curves. Note that a $Z^{\prime}$ that couples to electrons and muons with the same strength, as the case for the models studied here, can not explain the muon $g-2$ discrepancy [106].

Electroweak precision tests. Electroweak precision tests can also constrain $Z^{\prime}$ parameters. In figure 4 we present a constraint on $\epsilon$ taken from ref. [107]. In principle, similar constraints are present for all the $Z^{\prime}$ models, but need to be evaluated model-by-model, which is beyond the scope of our paper.

Loop-level meson and $Z$ decay limits. The $Z^{\prime}$ boson in the $\mathrm{U}(1)^{\prime}$ model could induce 1-loop level flavor-changing neutral current (FCNC) meson decays such as $B \rightarrow K+Z^{\prime}$ and $K \rightarrow \pi+Z^{\prime}$. The corresponding partial decay widths are not only directly relevant to the mass $m_{Z^{\prime}}$, gauge coupling $g^{\prime}$ (and also $\sin \theta$ ), but also depend subtly on the charges of the SM fermions under the $\mathrm{U}(1)^{\prime}$. For the $\mathrm{U}(1)_{B-L}$ case, all the SM mass and interaction terms are invariant under the $\mathrm{U}(1)_{B-L}$ gauge symmetry, and the widths $\Gamma\left(K \rightarrow \pi Z^{\prime}\right)$ and $\Gamma\left(B \rightarrow K Z^{\prime}\right)$ can be found e.g. in refs. [40, 108], which go to zero in the $m_{Z^{\prime}} \rightarrow 0$ limit, as expected from angular momentum conservation. As for the other $\mathrm{U}(1)^{\prime}$ and $Z-Z^{\prime}$ mixing models, the SM fermion couplings are explicitly chiral and so the fermion mass terms are not invariant under the $\mathrm{U}(1)^{\prime}$ gauge transformations. This leads to looplevel FCNC decays enhanced by the ratio $M^{2} / m_{Z^{\prime}}^{2}$ for a very light $Z^{\prime}$, with $M$ being the corresponding energy scale (typically the electroweak scale) for these decays [109-111]. Similarly, one can also have the 1-loop level decay $\Upsilon \rightarrow \gamma Z^{\prime}$ which is enhanced by $m_{b}^{2} / m_{Z^{\prime}}^{2}$ when $Z^{\prime}$ is light [112-114]. Thus the searches of $\Upsilon \rightarrow \gamma+$ inv. [115] and $\Upsilon \rightarrow \gamma+X$ with $X \rightarrow \mu^{+} \mu^{-}$[116] can be used to set limits on these $\mathrm{U}(1)^{\prime}$ models. If kinematically allowed, there is also the loop-level exotic $Z$ decay $Z \rightarrow \gamma Z^{\prime}$. The searches of single photon from $Z$ decay $[117,118]$ and $Z \rightarrow \gamma \ell^{+} \ell^{-}[119-121]$ at the LEP can be used to constrain the $Z^{\prime}$ bosons. However, these FCNC decay constraints become important only for sub$\mathrm{MeV}$ scale $Z^{\prime}$ bosons, and are not relevant for our P2 sensitivity analysis. Moreover, the $M^{2} / m_{Z}^{\prime 2}$-enhanced bounds cannot be arbitrarily strong in the $m_{Z^{\prime}} \rightarrow 0$ limit and one has to take the full momentum dependence into account in loop calculations. 
Astrophysical and cosmological limits. A sufficiently light $Z^{\prime}$ can also be constrained by astrophysical limits such as from supernova 1987A $[122,123]$ and cosmological limits such as from the big bang nucleosynthesis (BBN) [124, 125]. However, these limits mostly lie outside the window of parameter space considered in figures 2 to 6 . The BBN limits are significant only for $Z^{\prime}$ masses below $10 \mathrm{MeV}$, and the supernova limits apply additionally only for small couplings, see e.g. ref. [126]. Moreover, the BBN bound depends on the branching ratio of $Z^{\prime} \rightarrow \nu \bar{\nu}$ and $Z^{\prime} \rightarrow e^{-} e^{+}$. Thus these astrophysical and cosmological limits are not relevant to the P2 prospects, and thus are not shown in figures 2 to 6 .

\subsection{P2 sensitivities}

In this subsection, we derive the P2 sensitivities for the models considered in section 3 and compare them with the current experimental constraints discussed above.

\subsubsection{Chiral models}

The P2 sensitivities for the three chiral $\mathrm{U}(1)^{\prime}$ models given in table 2 are shown in figure 2 , where the $e+p$ sensitivities are shown as solid black lines, while the $e+{ }^{12} \mathrm{C}$ sensitivities are in dashed black lines. All existing relevant limits discussed in section 4.2 are presented in figure 2 as the shaded regions. Here we have assumed that there is no mass or kinetic $Z-Z^{\prime}$ mixing. It is clear from figure 2 that the limits for the three chiral $\mathrm{U}(1)^{\prime}$ models are rather similar: at low masses $m_{Z^{\prime}} \lesssim 100 \mathrm{GeV}$, the strongest limits are mainly from APV measurements (see below), beam-dump experiments, and from BaBar, NA48/2 and KLOE and $\mathrm{LHCb}$ data. For the models $\mathrm{U}(1)_{L}^{\prime}$ and $\mathrm{U}(1)_{X}^{\prime}$ the limits from $\nu+e$ scattering are also important, while for $\mathrm{U}(1)_{R}^{\prime}$ the $Z^{\prime}$ boson does not couple directly to neutrinos (see table 2 ), and as a result these are no neutrino scattering limits for the $\mathrm{U}(1)_{R}^{\prime}$ model. Suppressed by the charged lepton masses (see eq. (4.9)), the electron and muon $g-2$ limits are relatively weaker for these chiral models (and also for all other models in this paper). When $Z^{\prime}$ is heavier than the electroweak scale, the coupling $g^{\prime}$ is constrained by the direct searches of $Z^{\prime}$ in the LHC dijet data and the limits from the LEP $e^{+} e^{-} \rightarrow \ell^{+} \ell^{-}$data.

Comparing the solid and dashed black lines in the upper panel of figure 2, we see that the $e+{ }^{12} \mathrm{C}$ sensitivity for the $\mathrm{U}(1)_{L}^{\prime}$ model is more stringent than that from $e+p$ scattering by a factor of 1.4. This is mainly due to the enhancement of the effective coupling of $Z^{\prime}$ to the ${ }^{12} \mathrm{C}$ nucleus with respect to the proton. In particular, following eqs. (3.4) and (3.5), we have

$$
Q_{p}^{\prime}=\frac{1}{2}, \quad Q_{12 \mathrm{C}}^{\prime}=12 \quad \text { for } \mathrm{U}(1)_{L}^{\prime} .
$$

Taking into account the two different factors for $\Delta A^{\mathrm{PV}} / A_{\mathrm{SM}}^{\mathrm{PV}}$ in eq. (4.5), and the $\mathrm{SM}$ predictions of $A_{\mathrm{SM}}^{\mathrm{PV}}$ for proton and ${ }^{12} \mathrm{C}$ in eqs. (2.10) and (2.11), one gets the enhancement factor of 1.4. Similarly, as a result of

$$
Q_{p}^{\prime}=\frac{5}{4}, \quad Q_{12 \mathrm{C}}^{\prime}=18 \quad \text { for } \mathrm{U}(1)_{X}^{\prime},
$$

the $e+p$ and $e+{ }^{12} \mathrm{C}$ sensitivities for the $\mathrm{U}(1)_{X}^{\prime}$ model are almost the same, as can be seen in the bottom panel of figure 2 . For the $\mathrm{U}(1)_{R}^{\prime}$ model, due to the accidental cancellation of

$$
Q_{u_{L}}^{\prime}+Q_{u_{R}}^{\prime}+Q_{d_{L}}^{\prime}+Q_{d_{R}}^{\prime}=0
$$

we find that $Q_{{ }^{2} \mathrm{C}}^{\prime}=0$, and there is no $e+{ }^{12} \mathrm{C}$ sensitivity for the $\mathrm{U}(1)_{R}^{\prime}$ model. 


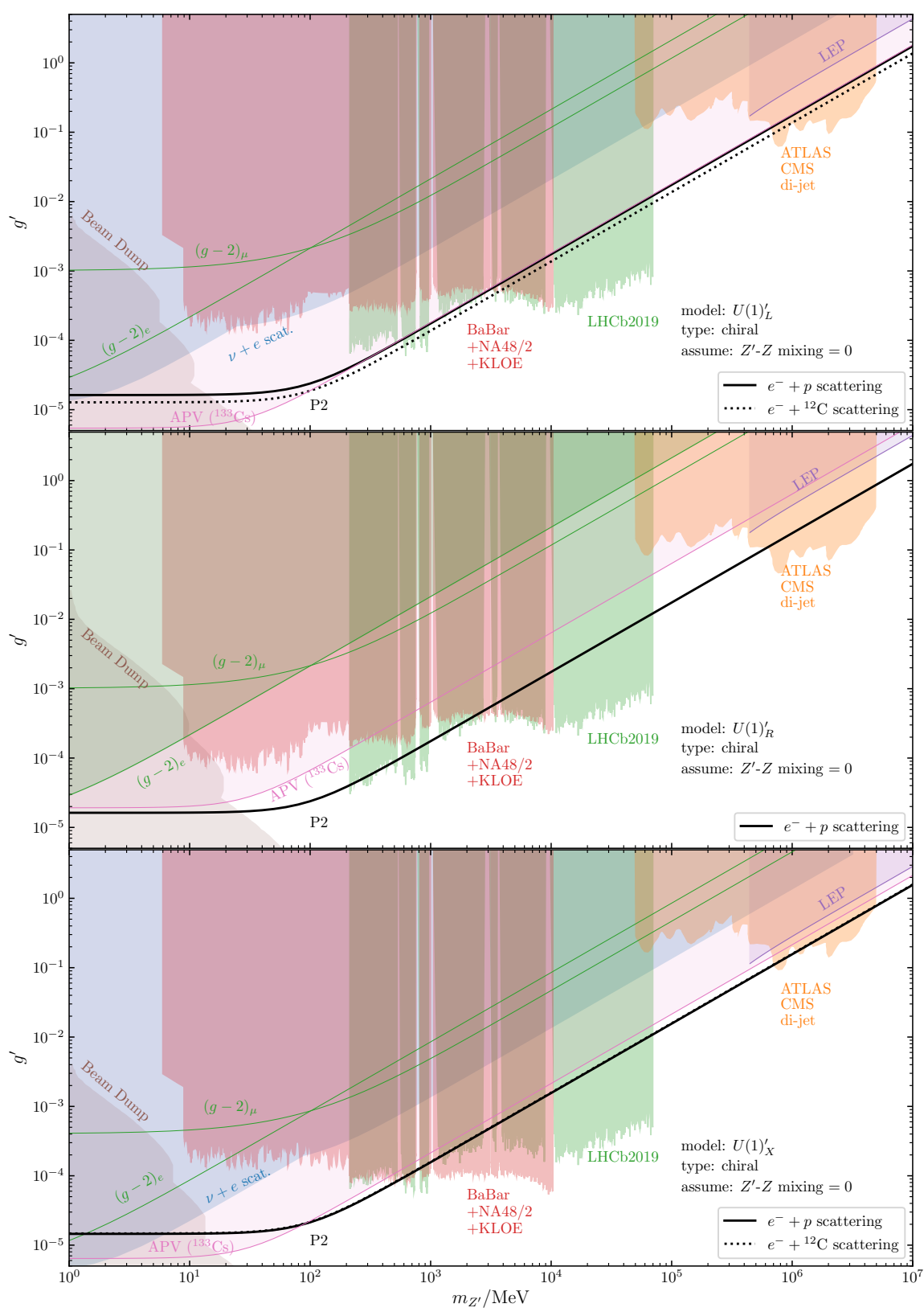

Figure 2. Sensitivities of the P2 experiment in $e+p$ (solid black) and $e+{ }^{12} \mathrm{C}$ (dashed black) scatterings to the gauge coupling $g^{\prime}$ in three chiral $\mathrm{U}(1)^{\prime}$ models given in table 2 . For simplicity we have assumed here that there is no mass or kinetic $Z-Z^{\prime}$ mixing. The shaded regions are excluded by APV measurements [24-27], beam-dump experiments [74-77], electron and muon $g-2$ [1, 102], BaBar [79, 80], NA48/2 [81], KLOE [82], LHCb [84], LEP $e^{+} e^{-} \rightarrow \ell^{+} \ell^{-}$data [89] and the ATLAS and CMS dijet data [90]. Note that, due to the cancellation of quantum numbers in eq. (3.5), the $\mathrm{P} 2$ prospect in the $e+{ }^{12} \mathrm{C}$ channel is absent for the $\mathrm{U}(1)_{R}^{\prime}$ model (middle panel). See sections 4.2 and 4.3 for more details. 


\begin{tabular}{|c|c|c|c|c|}
\hline \multirow{2}{*}{ models } & \multicolumn{2}{|c|}{$m_{Z^{\prime}} \rightarrow 0$} & \multicolumn{2}{c|}{$m_{Z^{\prime}} \rightarrow \infty$} \\
\cline { 2 - 5 } & $e+p$ & $e+{ }^{12} \mathrm{C}$ & $e+p$ & $e+{ }^{12} \mathrm{C}$ \\
\hline $\mathrm{U}(1)_{L}^{\prime}$ & $g^{\prime}<1.7 \times 10^{-5}$ & $g^{\prime}<1.2 \times 10^{-5}$ & $\frac{m_{Z^{\prime}}}{g^{\prime}}>5.9 \mathrm{TeV}$ & $\frac{m_{Z^{\prime}}}{g^{\prime}}>8.3 \mathrm{TeV}$ \\
\hline $\mathrm{U}(1)_{R}^{\prime}$ & $g^{\prime}<1.7 \times 10^{-5}$ & - & $\frac{m_{Z^{\prime}}}{g^{\prime}}>5.6 \mathrm{TeV}$ & - \\
\hline $\mathrm{U}(1)_{X}^{\prime}$ & $g^{\prime}<1.5 \times 10^{-5}$ & $g^{\prime}<1.5 \times 10^{-5}$ & $\frac{m_{Z^{\prime}}}{g^{\prime}}>7.7 \mathrm{TeV}$ & $\frac{m_{Z^{\prime}}}{g^{\prime}}>7.7 \mathrm{TeV}$ \\
\hline $\mathrm{U}(1)^{\prime}(\sin \theta)$ & $\sin \theta<1.7 \times 10^{-4}$ & $\sin \theta<7.3 \times 10^{-5}$ & $\sin \theta<0.13$ & $\sin \theta<0.07$ \\
\hline $\mathrm{U}(1)^{\prime}(\epsilon)$ & $\epsilon<0.05$ & $\epsilon<0.07$ & $\frac{m_{Z^{\prime}}}{\epsilon}>350 \mathrm{GeV}$ & $\frac{m_{Z^{\prime}}}{\epsilon}>600 \mathrm{GeV}$ \\
\hline $\mathrm{U}(1)_{B}(0.01)$ & $\sin \theta<9 \times 10^{-5}$ & $\sin \theta<9 \times 10^{-5}$ & $\sin \theta<0.13$ & $\sin \theta<0.07$ \\
\hline $\mathrm{U}(1)_{B}(1)$ & $\sin \theta<1.1 \times 10^{-5}$ & $\sin \theta<1.2 \times 10^{-5}$ & $\sin \theta<0.13$ & $\sin \theta<0.07$ \\
\hline $\mathrm{U}(1)_{B}(10)$ & $\sin \theta<3.7 \times 10^{-6}$ & $\sin \theta<4.1 \times 10^{-6}$ & $\sin \theta<0.13$ & $\sin \theta<0.07$ \\
\hline $\mathrm{U}(1)_{B-L}(0.01)$ & $\sin \theta<2.9 \times 10^{-4}$ & $\sin \theta<6.9 \times 10^{-5}$ & $\sin \theta<0.13$ & $\sin \theta<0.07$ \\
\hline $\mathrm{U}(1)_{B-L}(1)$ & $\sin \theta<2 \times 10^{-5}$ & $\sin \theta<2 \times 10^{-5}$ & $\sin \theta<0.13$ & $\sin \theta<0.07$ \\
\hline $\mathrm{U}(1)_{B-L}(10)$ & $\sin \theta<6 \times 10^{-6}$ & $\sin \theta<6.5 \times 10^{-6}$ & $\sin \theta<0.13$ & $\sin \theta<0.07$ \\
\hline
\end{tabular}

Table 3. P2 sensitivities for the chiral and non-chiral models shown in figures 2 to 6 in the limits of $m_{Z^{\prime}} \rightarrow 0$ and $m_{Z^{\prime}} \rightarrow \infty$. "U(1) (sin $\left.\theta, \epsilon\right)$ " refers to the generic $\mathrm{U}(1)^{\prime}$ model with only mass mixing $\sin \theta$ or kinetic mixing $\epsilon$. The numbers in the parentheses for the $\mathrm{U}(1)_{B}$ and $\mathrm{U}(1)_{B-L}$ models are the values of $g^{\prime} / \sin \theta$. Note that for the $\mathrm{U}(1)_{R}^{\prime}$ model there is no sensitivity in the $e+{ }^{12} \mathrm{C}$ scattering mode due to an accidental cancellation (cf. eq. (4.15)).

For masses $m_{Z^{\prime}} \lesssim 100 \mathrm{MeV}$, the $\mathrm{P} 2$ sensitivities are almost independent of the $Z^{\prime}$ mass, which correspond to the plateau in the three panels of figure 2 . This is also true for the other models considered here (see figures 3 to 6 ). In the high $m_{Z^{\prime}}$ limit, i.e. $m_{Z^{\prime}} \rightarrow \infty$, we can safely neglect the momentum transfer $Q^{2}$, and the P2 experiment can probe an effective ultraviolet (UV) cutoff scale of $\Lambda=m_{Z^{\prime}} / g^{\prime}$ at the few-TeV scale. All the P2 sensitivities in the limits of $m_{Z^{\prime}} \rightarrow 0$ and $m_{Z^{\prime}} \rightarrow \infty$ for the three chiral models are collected in table 3 .

The low-energy nuclear transition in ${ }^{133} \mathrm{Cs}$ is essentially equivalent to electron-nucleus scattering, thus for all our cases the properties of the APV limits are very similar to those for the P2 prospects. Comparing the ${ }^{133} \mathrm{Cs}$ atom in the most precise APV measurement with ${ }^{12} \mathrm{C}$ in $\mathrm{P} 2$, the differences lie mainly in the following factors: the nuclear weak charges, the effective couplings of $Z^{\prime}$ to nuclei and the energy scale $Q$. Taking the $\mathrm{U}(1)_{L}^{\prime}$ model as an explicit example, the coupling of $Z^{\prime}$ to ${ }^{133} \mathrm{Cs}$ is

$$
Q_{133 \mathrm{Cs}}^{\prime}=\frac{1}{2}\left(266 Q_{u_{L}}^{\prime}+266 Q_{u_{R}}^{\prime}+211 Q_{d_{L}}^{\prime}+211 Q_{d_{R}}^{\prime}\right)=\frac{263}{2}
$$

Following eq. (3.3), the resulting parity violation for the atom $X$ with respect to the SM value is proportional to

$$
\frac{\Delta A^{\mathrm{PV}}(X)}{A_{\mathrm{SM}}^{\mathrm{PV}}} \propto Q^{-2} \frac{Q_{X}^{\prime}}{Q_{W}(X)} \frac{Q^{2}}{Q^{2}+m_{Z^{\prime}}^{2}} .
$$




\begin{tabular}{|c|c|c|c|}
\hline models & mode & $m_{Z^{\prime}}$ ranges & $g^{\prime}$ ranges \\
\hline \multirow{4}{*}{$\mathrm{U}(1)_{L}^{\prime}$} & $e+p$ & - & - \\
\hline & & {$[100 \mathrm{MeV}, 200 \mathrm{MeV}]$} & {$\left[2.0 \times 10^{-5}, 3.3 \times 10^{-5}\right]$} \\
\hline & $e+{ }^{12} \mathrm{C}$ & {$[70 \mathrm{GeV}, 600 \mathrm{GeV}]$} & {$[0.01,0.09]$} \\
\hline & & {$[5 \mathrm{TeV}, 90 \mathrm{TeV}]$} & {$[0.70,4 \pi]$} \\
\hline \multirow{3}{*}{$\mathrm{U}(1)_{R}^{\prime}$} & & {$[20 \mathrm{MeV}, 200 \mathrm{MeV}]$} & {$\left[1.7 \times 10^{-5}, 4.0 \times 10^{-5}\right]$} \\
\hline & $e+p$ & {$[70 \mathrm{GeV}, 600 \mathrm{GeV}]$} & {$[0.012,0.1]$} \\
\hline & & {$[5 \mathrm{TeV}, 70 \mathrm{TeV}]$} & {$[0.9,4 \pi]$} \\
\hline \multirow{6}{*}{$\mathrm{U}(1)_{X}^{\prime}$} & & {$[93 \mathrm{MeV}, 430 \mathrm{MeV}]$} & {$\left[2.0 \times 10^{-5}, 7.5 \times 10^{-5}\right]$} \\
\hline & $e+p$ & {$[70 \mathrm{GeV}, 700 \mathrm{GeV}]$} & {$[0.01,0.11]$} \\
\hline & & {$[5 \mathrm{TeV}, 79 \mathrm{TeV}]$} & {$[0.8,4 \pi]$} \\
\hline & & {$[93 \mathrm{MeV}, 430 \mathrm{MeV}]$} & {$\left[2.0 \times 10^{-5}, 7.5 \times 10^{-5}\right]$} \\
\hline & $e+{ }^{12} \mathrm{C}$ & {$[70 \mathrm{GeV}, 700 \mathrm{GeV}]$} & {$[0.01,0.11]$} \\
\hline & & {$[5 \mathrm{TeV}, 79 \mathrm{TeV}]$} & {$[0.8,4 \pi]$} \\
\hline
\end{tabular}

Table 4. Ranges of $m_{Z^{\prime}}$ and $g^{\prime}$ where P2 will be able to improve current limits for chiral U(1) models. This table is a summary of figure 2. The columns of $\mathrm{U}(1)_{L}^{\prime}$ in the $e+p$ mode are void because P2 does not have sensitivity beyond existing APV limits.

In the limit of $m_{Z^{\prime}} \ll Q$, we can neglect the last factor in the equation above, and compare the APV sensitivities in ${ }^{133} \mathrm{Cs}$ and the $\mathrm{P} 2$ prospects using ${ }^{12} \mathrm{C}$ :

$$
\begin{aligned}
\frac{\Delta A^{\mathrm{PV}}\left({ }^{133} \mathrm{Cs}\right) / A_{\mathrm{SM}}^{\mathrm{PV}}\left({ }^{133} \mathrm{Cs}\right)}{\Delta A^{\mathrm{PV}}\left({ }^{12} \mathrm{C}\right) / A_{\mathrm{SM}}^{\mathrm{PV}}\left({ }^{12} \mathrm{C}\right)}\left(m_{Z^{\prime}} \ll Q\right) & =\left(\frac{Q_{\mathrm{P} 2}}{Q_{\mathrm{APV}}}\right)^{2} \frac{Q_{133}^{\prime} \mathrm{Cs} / Q_{W}\left({ }^{133} \mathrm{Cs}\right)}{Q_{12}^{\prime} / Q_{W}\left({ }^{12} \mathrm{C}\right)} \\
& \simeq\left(\frac{93 \mathrm{MeV}}{30 \mathrm{MeV}}\right)^{2}\left(\frac{-1.78}{-2.17}\right) .
\end{aligned}
$$

Taking into account the difference of the APV accuracy $(0.77 \%)$ and the P2 precision in ${ }^{12} \mathrm{C}(0.49 \%)$, eq. (4.18) implies that for the $\mathrm{U}(1)^{\prime}$ model at low energies $m_{Z^{\prime}} \ll Q$, the APV limit on $g^{\prime}$ is more stringent than the P2 prospect using ${ }^{12} \mathrm{C}$ by a factor of

$$
\left(\frac{0.49 \%}{0.77 \%}\right)^{1 / 2}\left(\frac{93 \mathrm{MeV}}{30 \mathrm{MeV}}\right)\left(\frac{-1.78}{-2.17}\right)^{1 / 2} \simeq 2.24
$$

In the limit of $m_{Z^{\prime}} \gg Q$, the last factor in eq. (4.17) can be simplified to $Q^{2} / m_{Z^{\prime}}$ and any momentum dependence cancels. In this case the comparison of APV and P2 with the target ${ }^{12} \mathrm{C}$ is determined only by the coupling factors $Q_{N}^{\prime}$ and $Q_{W}$, i.e.

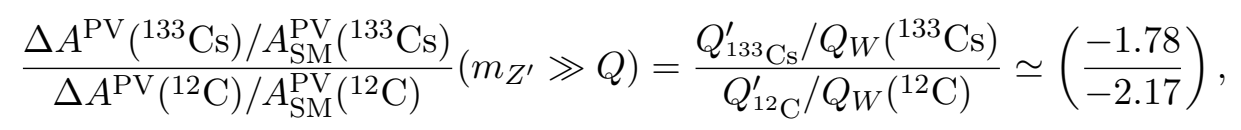


and the APV limit on $g^{\prime}$ is weaker than the $\mathrm{P} 2$ prospect with ${ }^{12} \mathrm{C}$ by a factor of

$$
\left(\frac{0.49 \%}{0.77 \%}\right)^{1 / 2}\left(\frac{-1.78}{-2.17}\right)^{1 / 2} \simeq 0.73
$$

as shown in the upper panel of figure 2. In a similar way, one can compare the APV limits with the P2 sensitivities for the proton target for the $\mathrm{U}(1)^{\prime}$ models. It turns out that for the $\mathrm{U}(1)_{L}^{\prime}$ model the APV constraint is almost the same as that for the P2 prospect with the proton target in the limit of large $m_{Z^{\prime}}$. For the $\mathrm{U}(1)_{R}^{\prime}$ model, the APV limit is slightly weaker than the $\mathrm{P} 2$ sensitivity with protons for all values of $m_{Z^{\prime}}$. For the $\mathrm{U}(1)_{X}^{\prime}$ model the APV limits exclude the P2 prospects at the low mass range of $m_{Z^{\prime}}$, and are slightly weaker than the $\mathrm{P} 2$ sensitivities for both proton and ${ }^{12} \mathrm{C}$ when the $Z^{\prime}$ mass is large.

The P2 sensitivities in the lower mass range have also been precluded partially by the beam-dump experiments, NA62, Na48/2 and KLOE experiments, and in the high mass range partially by the LHC dijet data. However, even if all these constraints are taken into consideration, there is still some parameter space in the $m_{Z^{\prime}} g^{\prime}$ plane that can be probed at the $\mathrm{P} 2$ experiment, which is collected in table 4. Note that the P2 prospects for the $\mathrm{U}(1)_{L}^{\prime}$ model in the proton mode have been precluded by the APV limits. Depending on the U(1) model, $Z^{\prime}$ boson masses can be probed down to roughly $20 \mathrm{MeV}$, and the coupling $g^{\prime}$ can be probed down to the order of $10^{-5}$. In the high $Z^{\prime}$ mass end, if the gauge coupling is fixed at the perturbative limit of $4 \pi$, the $\mathrm{P} 2$ experiment can reach $Z^{\prime}$ masses up to $70 \mathrm{TeV}$ and $79 \mathrm{TeV}$ for the chiral $\mathrm{U}(1)_{R}^{\prime}$ and $\mathrm{U}(1)_{X}^{\prime}$ models respectively in $e+p$ scattering, and up to $90 \mathrm{TeV}$ and $79 \mathrm{TeV}$ for the $\mathrm{U}(1)_{L}^{\prime}$ and $\mathrm{U}(1)_{X}^{\prime}$ models in $e+{ }^{12} \mathrm{C}$ mode. The P2 sensitivities of heavy $Z^{\prime}$ bosons are largely complementary to the direct searches at the LHC and future higher energy colliders.

\subsubsection{Non-chiral models}

For the generic non-chiral $\mathrm{U}(1)^{\prime}$ model with $Z-Z^{\prime}$ mass mixing, the $\mathrm{P} 2$ sensitivities and corresponding existing limits are shown in figure 3 for the simplest case of $g^{\prime} Q^{\prime}=0$. This corresponds to the cases of negligibly small couplings $\left(g^{\prime} / \sin \theta \ll 1\right)$ or no SM fermions carrying $\mathrm{U}(1)^{\prime}$ charges (known as the secluded $Z^{\prime}$ model). The limits from LEP $e^{+} e^{-} \rightarrow \ell^{+} \ell^{-}$ data are very weak and thus are not shown in figure 3 . The P2 sensitivity for this model is given in eq. (3.13). As in figure 2 , for $m_{Z^{\prime}} \lesssim 100 \mathrm{MeV}$, the P2 sensitivities are almost independent of $m_{Z^{\prime}}$. In the mass range $100 \mathrm{MeV} \lesssim m_{Z^{\prime}} \lesssim m_{Z}$, the factor in the parentheses of eq. (3.13) can be simplified to be

$$
\frac{m_{Z}^{2}}{m_{Z^{\prime}}^{2}+Q^{2}}-1 \simeq \frac{m_{Z}^{2}}{m_{Z^{\prime}}^{2}} .
$$

Therefore, in this mass range P2 excludes an effective cutoff scale $\Lambda=m_{Z^{\prime}} / \sin \theta$, which is $560 \mathrm{GeV}$ and $1.1 \mathrm{TeV}$ for the $e+p$ and $e+{ }^{12} \mathrm{C}$ scattering, respectively. The P2 sensitivity vanishes when the $Z^{\prime}$ mass is close to $m_{Z}$. When $m_{Z^{\prime}}>m_{Z}$, the factor in eq. (4.22) changes its sign, and approaches the value -1 in the limit of $m_{Z^{\prime}} \gg m_{Z}$. As a result, the P2 sensitivities are constant in the heavy $Z^{\prime}$ limits, which are respectively $\sin \theta<0.13$ and 0.07 for $e+p$ and $e+{ }^{12} \mathrm{C}$ scattering. 


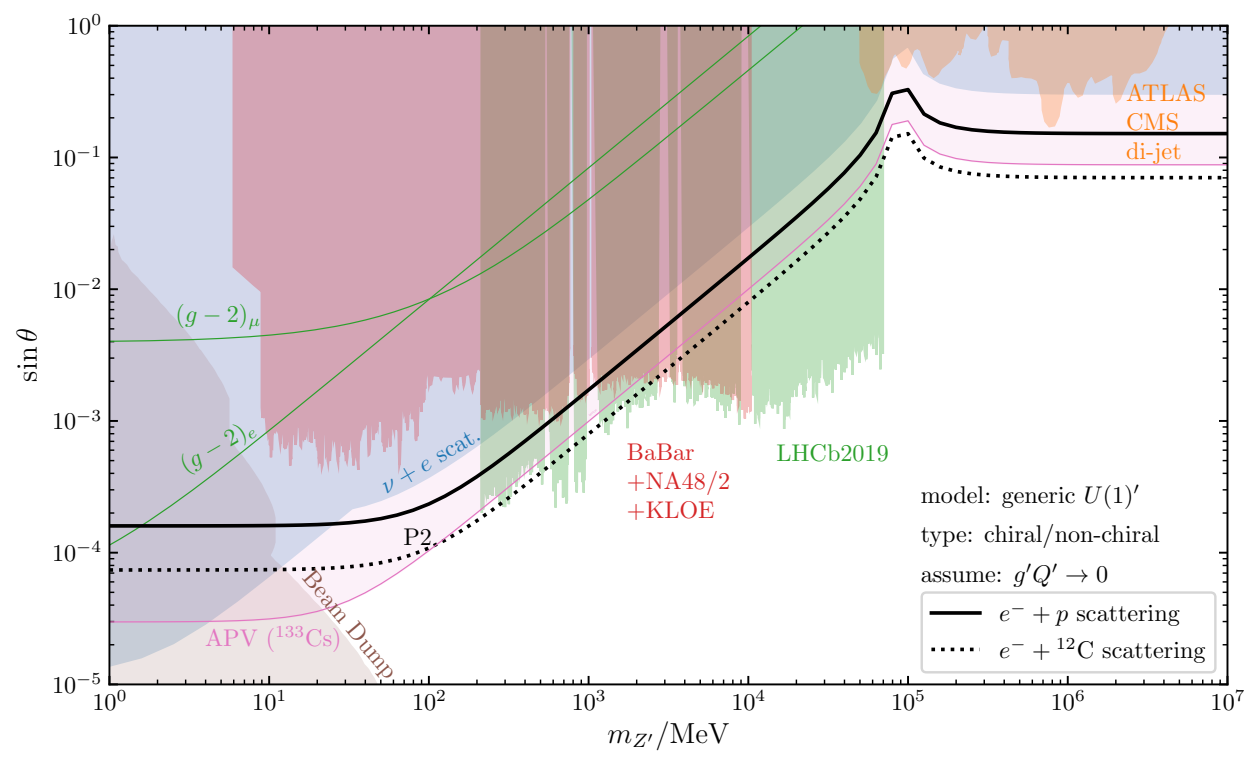

Figure 3. Sensitivity of the $\mathrm{P} 2$ experiment to the generic $\mathrm{U}(1)^{\prime}$ model with only mass mixing $\sin \theta$ and in the limit of $g^{\prime} Q^{\prime}=0$. The notation is the same as in figure 2 .

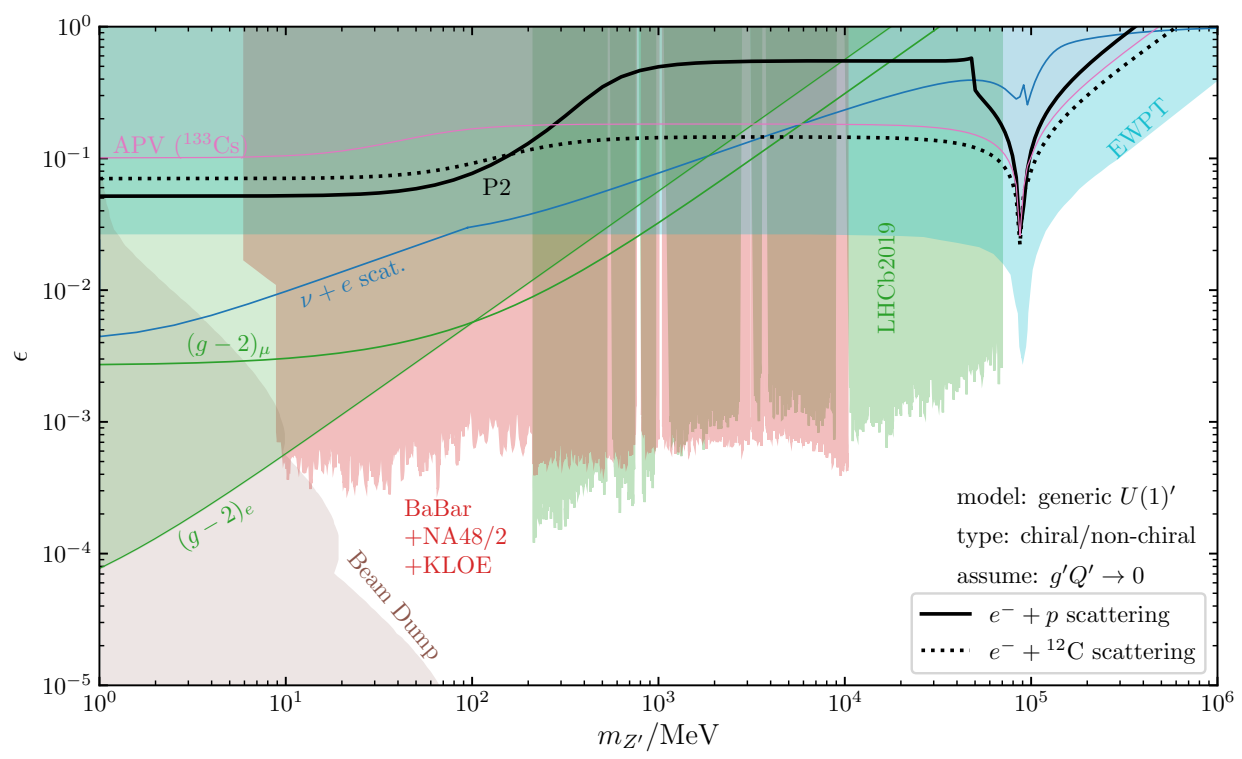

Figure 4. Sensitivity of the $\mathrm{P} 2$ experiment to the generic $\mathrm{U}(1)^{\prime}$ model with only kinetic mixing $\epsilon$ and in the limit of $g^{\prime} Q^{\prime}=0$. The notation is the same as in figure 2 . 


\begin{tabular}{|c|c|c|c|}
\hline models & mode & $m_{Z^{\prime}}$ ranges & $\sin \theta$ or $\epsilon$ ranges \\
\hline \multirow{2}{*}{$\mathrm{U}(1)^{\prime}(\sin \theta)$} & $e+p$ & - & - \\
\hline & $e+{ }^{12} \mathrm{C}$ & $\begin{array}{c}{[110 \mathrm{MeV}, 200 \mathrm{MeV}]} \\
(>70 \mathrm{GeV})\end{array}$ & $\begin{array}{c}{\left[1.1 \times 10^{-4}, 1.9 \times 10^{-4}\right]} \\
{[0.07,0.15]}\end{array}$ \\
\hline \multirow{2}{*}{$\mathrm{U}(1)^{\prime}(\epsilon)$} & $e+p$ & - & - \\
\hline & $e+{ }^{12} \mathrm{C}$ & - & - \\
\hline \multirow[t]{2}{*}{$\mathrm{U}(1)_{B}(0.01)$} & $e+p$ & $\begin{array}{c}{[120 \mathrm{MeV}, 200 \mathrm{MeV}]} \\
{[70 \mathrm{GeV}, 100 \mathrm{GeV}]}\end{array}$ & $\begin{array}{c}{\left[1.4 \times 10^{-4}, 2.3 \times 10^{-4}\right]} \\
{[0.08,0.20]}\end{array}$ \\
\hline & $e+{ }^{12} \mathrm{C}$ & $\begin{array}{c}{[120 \mathrm{MeV}, 200 \mathrm{MeV}]} \\
(>70 \mathrm{GeV})\end{array}$ & $\begin{array}{c}{\left[1.4 \times 10^{-4}, 2.3 \times 10^{-4}\right]} \\
{[0.07,0.13]}\end{array}$ \\
\hline \multirow[t]{2}{*}{$\mathrm{U}(1)_{B}(1)$} & $e+p$ & $\begin{array}{c}{[70 \mathrm{MeV}, 10 \mathrm{GeV}]} \\
{[70 \mathrm{GeV}, 500 \mathrm{GeV}]}\end{array}$ & $\begin{array}{c}{\left[1.2 \times 10^{-5}, 1.2 \times 10^{-3}\right]} \\
{\left[8 \times 10^{-3}, 0.06\right]}\end{array}$ \\
\hline & $e+{ }^{12} \mathrm{C}$ & $\begin{array}{c}{[83 \mathrm{MeV}, 10 \mathrm{GeV}]} \\
{[70 \mathrm{GeV}, 650 \mathrm{GeV}]} \\
\quad(>2.3 \mathrm{TeV})\end{array}$ & $\begin{array}{c}{\left[1.4 \times 10^{-5}, 1.3 \times 10^{-3}\right]} \\
{\left[9 \times 10^{-3}, 0.055\right]} \\
{[0.067,0.07]}\end{array}$ \\
\hline \multirow{2}{*}{$\mathrm{U}(1)_{B}(10)$} & $e+p$ & {$[70 \mathrm{MeV}, 305 \mathrm{GeV}]$} & {$\left[4.0 \times 10^{-6}, 0.012\right]$} \\
\hline & $e+{ }^{12} \mathrm{C}$ & $\begin{array}{c}{[83 \mathrm{MeV}, 305 \mathrm{GeV}]} \\
(>5 \mathrm{TeV})\end{array}$ & $\begin{array}{c}{\left[4.2 \times 10^{-6}, 0.012\right]} \\
{[0.067,0.07]}\end{array}$ \\
\hline \multirow{2}{*}{$\mathrm{U}(1)_{B-L}(0.01)$} & $e+p$ & {$[90 \mathrm{GeV}, 110 \mathrm{GeV}]$} & {$[0.17,0.19]$} \\
\hline & $e+{ }^{12} \mathrm{C}$ & $\begin{array}{c}{[110 \mathrm{MeV}, 560 \mathrm{MeV}]} \\
(>70 \mathrm{GeV})\end{array}$ & $\begin{array}{c}{\left[1 \times 10^{-4}, 5.0 \times 10^{-4}\right]} \\
{[0.07,0.2]}\end{array}$ \\
\hline \multirow[t]{2}{*}{$\mathrm{U}(1)_{B-L}(1)$} & $e+p$ & $\begin{array}{l}{[80 \mathrm{MeV}, 600 \mathrm{MeV}]} \\
{[70 \mathrm{GeV}, 620 \mathrm{GeV}]}\end{array}$ & $\begin{array}{c}{\left[2.4 \times 10^{-5}, 1.2 \times 10^{-4}\right]} \\
{[0.013,0.1]}\end{array}$ \\
\hline & $e+{ }^{12} \mathrm{C}$ & $\begin{array}{c}{[80 \mathrm{MeV}, 600 \mathrm{MeV}]} \\
{[70 \mathrm{GeV}, 260 \mathrm{GeV}]} \\
\quad(>400 \mathrm{GeV})\end{array}$ & $\begin{array}{c}{\left[2.4 \times 10^{-5}, 1.2 \times 10^{-4}\right]} \\
{[0.013,0.18]}\end{array}$ \\
\hline \multirow[t]{2}{*}{$\mathrm{U}(1)_{B-L}(10)$} & $e+p$ & $\begin{array}{l}{[70 \mathrm{MeV}, 200 \mathrm{MeV}]} \\
{[70 \mathrm{GeV}, 420 \mathrm{GeV}]}\end{array}$ & $\begin{array}{c}{\left[7.0 \times 10^{-6}, 1.4 \times 10^{-5}\right]} \\
{\left[4.3 \times 10^{-3}, 0.028\right]}\end{array}$ \\
\hline & $e+{ }^{12} \mathrm{C}$ & $\begin{array}{l}{[83 \mathrm{MeV}, 600 \mathrm{MeV}]} \\
{[70 \mathrm{GeV}, 420 \mathrm{GeV}]}\end{array}$ & $\begin{array}{c}{\left[9.0 \times 10^{-6}, 1.5 \times 10^{-5}\right]} \\
{\left[4.5 \times 10^{-3}, 0.032\right]}\end{array}$ \\
\hline
\end{tabular}

Table 5. Similar to table 4 except for non-chiral U(1)' models, summarized from figures 3 to 6 . The columns of $\mathrm{U}(1)^{\prime}(\sin \theta)$ in the $e+p$ mode and $\mathrm{U}(1)^{\prime}(\epsilon)$ for all models are void because $\mathrm{P} 2$ does not have better sensitivities than the existing limits. 
Similar to the chiral models, the APV limits in the generic $\mathrm{U}(1)^{\prime}$ model have the same features as those for the $\mathrm{P} 2$ prospects. In the limit of $m_{Z^{\prime}} \ll Q$, eq. (3.13) implies that the new physics contributions to APV is mostly determined by the energy scale $Q$, i.e.

$$
\frac{\Delta A^{\mathrm{PV}}\left({ }^{133} \mathrm{Cs}\right) / A_{\mathrm{SM}}^{\mathrm{PV}}\left({ }^{133} \mathrm{Cs}\right)}{\Delta A^{\mathrm{PV}}\left({ }^{12} \mathrm{C}\right) / A_{\mathrm{SM}}^{\mathrm{PV}}\left({ }^{12} \mathrm{C}\right)}\left(m_{Z^{\prime}} \ll Q\right)=\left(\frac{93 \mathrm{MeV}}{30 \mathrm{MeV}}\right)^{2},
$$

and the resulting APV limit is stronger than the P2 prospect in $e+{ }^{12} \mathrm{C}$ scattering by a factor of

$$
\left(\frac{0.49 \%}{0.77 \%}\right)^{1 / 2}\left(\frac{93 \mathrm{MeV}}{30 \mathrm{MeV}}\right) \simeq 2.47 .
$$

In the limit of $m_{Z^{\prime}} \gg Q$, comparison of the APV limits and the P2 prospects will be only dictated by the accuracies, i.e. the APV limit is weaker than the P2 sensitivity for $e+{ }^{12} \mathrm{C}$ by a factor of $\sqrt{0.49 \% / 0.77 \%} \simeq 0.80$. As shown in figure 3 , the APV constraints have excluded P2 sensitivities for $e+p$ scattering. The limit from $\nu+e$ scattering has the same feature in the high $Z^{\prime}$ mass range, and can go to masses beyond the ones from direct LHC searches, as shown in figure 3. However, the P2 experiment can exceed the neutrino scattering limits. The resultant $\mathrm{P} 2$ ranges of $m_{Z^{\prime}}$ and $\sin \theta$ are collected in table 5 .

The P2 sensitivities for the most generic $\mathrm{U}(1)^{\prime}$ model with a kinetic mixing of $Z^{\prime}$ with the SM $Z$ boson and with $g^{\prime} Q^{\prime}=0$, as well as the corresponding existing limits, are presented in figure 4. As shown in this figure, the P2 sensitivities for this case are not as competitive as others. This is because $Z^{\prime}$ with a sizable kinetic mixing is more photonlike when $m_{Z^{\prime}}$ decreases. In particular, based on the couplings in table 1 , in the limit of $m_{Z^{\prime}} \rightarrow 0$

$$
g_{e_{L}}^{\prime}-g_{e_{R}}^{\prime} \propto \frac{m_{Z^{\prime}}^{2}}{m_{Z}^{2}} \rightarrow 0 .
$$

In fact, all the $g_{f}^{\prime}$ couplings are proportional to the electric charge of $f$ in this limit, which implies that $Z^{\prime}$ would not mediate parity-violating processes. In this case, $\epsilon$ is mainly constrained by the modification of $Z$ couplings. As a result of the enhancement of $\Delta A^{\mathrm{PV}}$ due to the $\left(1-r_{m}\right)$ factor in the denominator of eqs. (3.14) and (3.15), there are dips at the $Z$ mass. However, the P2 sensitivities at the high $Z^{\prime}$ mass range are still precluded by the electroweak precision data.

The P2 sensitivities for the $\mathrm{U}(1)_{B}$ model with $Z-Z^{\prime}$ mass mixing and three values of $g^{\prime} / \sin \theta=0.01,1$ and 10 are shown respectively in the upper, middle and lower panels of figure 5. The master formula for $\Delta A^{\mathrm{PV}}$ is given in eq. (3.16). For the three benchmark values, the $R$ ratio is respectively 1.96, 196 and 1960 for $e+p$ scattering, and $-0.34,-34$ and -340 for $e+{ }^{12} \mathrm{C}$ scattering. Generally speaking, when the $Z^{\prime}$ mass is small such that $m_{Z}^{2} \gtrsim m_{Z^{\prime}}^{2}+Q^{2}$, the P2 sensitivities of $\sin \theta$ can be significantly improved by a factor of $\sqrt{1+R}$. For sufficiently large $m_{Z^{\prime}}$, i.e.

$$
m_{Z^{\prime}}=\sqrt{1+R} m_{Z},
$$

the two terms in eq. (3.16) cancel each other for all three values of $g^{\prime}$ in $e+p$ scattering, which turns out to happen respectively at

$$
m_{Z^{\prime}}=1.72 m_{Z}[157 \mathrm{GeV}], \quad 14.1 m_{Z^{\prime}}[1.28 \mathrm{TeV}], \quad 44.3 m_{Z^{\prime}} \quad[4.03 \mathrm{TeV}]
$$



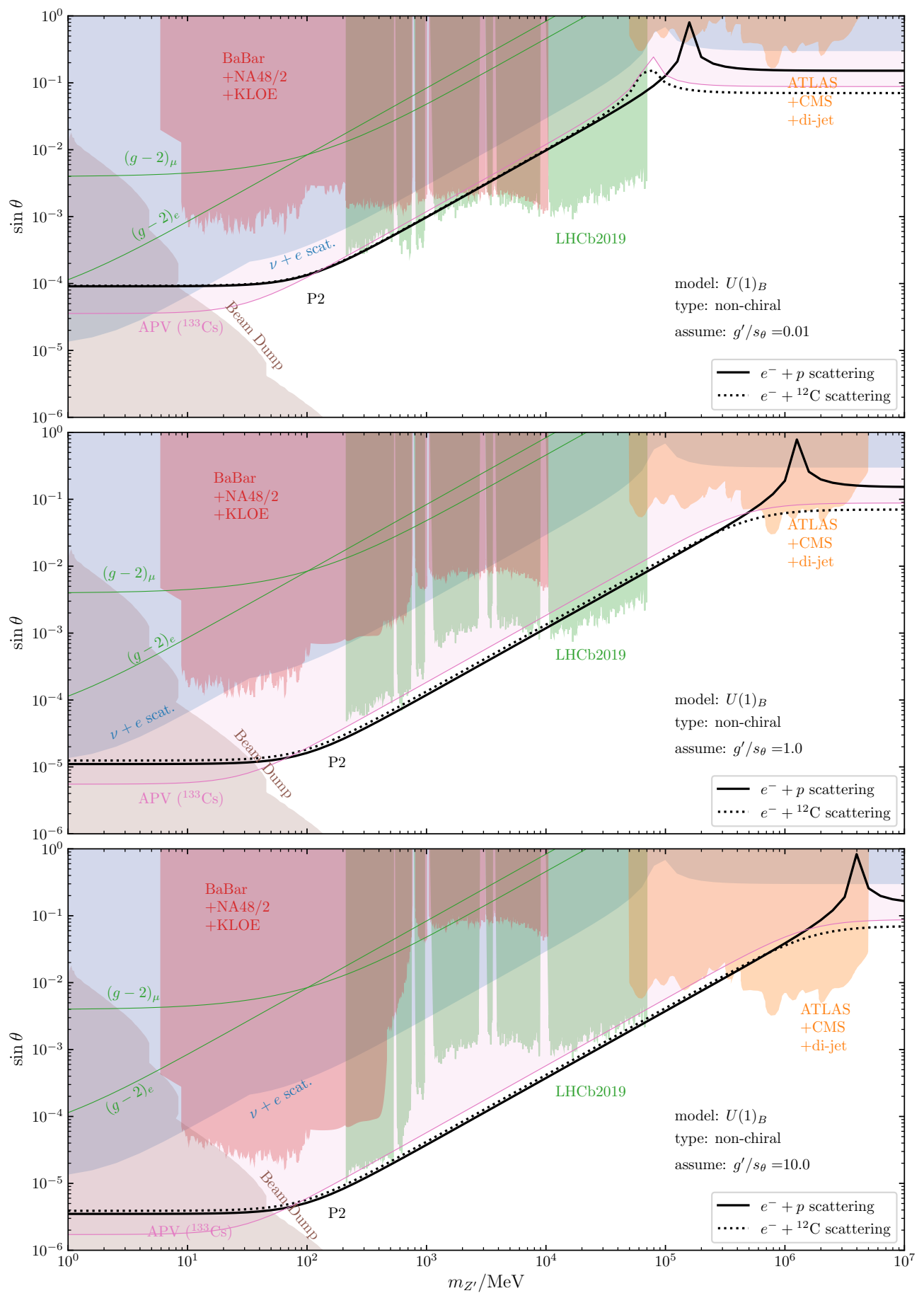

Figure 5. Sensitivity of the $\mathrm{P} 2$ experiment to the chiral $\mathrm{U}(1)_{B}$ model with mass mixing $\sin \theta$ and the three values of $g^{\prime} / \sin \theta=0.01$ (upper), 1 (middle) and 10 (lower). The notations are the same as in figure 2 . 
as indicated by the solid black peaks in figure 5. For the case of $g^{\prime} / \sin \theta=0.01$ in $e+{ }^{12} \mathrm{C}$ scattering, the cancellation happens at

$$
m_{Z^{\prime}}=0.81 m_{Z}[74 \mathrm{GeV}]
$$

as shown by the peak of the dashed black line in the upper panel of figure 5. For the two cases of $g^{\prime} / \sin \theta=1$ and 10 in $e+{ }^{12} \mathrm{C}$ scattering, the factor $R$ is below -1 and there is no cancellation between the two terms in eq. (3.16). Therefore there are no peaks for the dashed curves in the middle and lower panels of figure 5 . In the limit of $m_{Z^{\prime}} \gg \sqrt{1+R} m_{Z}$, the first term in the bracket of eq. (3.16) can be neglected, and the P2 sensitivity to $\sin \theta$ approaches a constant value, which is the same as in figure 3 . For the case of $g^{\prime} / \sin \theta=0.01$, the current limits on the mass mixing angle $\sin \theta$ in the upper panel of figure 5 are almost the same as in figure 3 , while the limits for the cases of $g / \sin \theta=1$ and 10 with larger gauge couplings tend to be more stringent, as presented in the middle and lower panels of figure 5. In light of all the limits, the ranges of $m_{Z^{\prime}}$ and $\sin \theta$ that can be probed at P2 for the $\mathrm{U}(1)_{B}$ model are collected in table 5 .

The $\mathrm{U}(1)_{B-L}$ model with $Z-Z^{\prime}$ mass mixing is quite similar to the $\mathrm{U}(1)_{B}$ model, and the corresponding P2 sensitivities and current limits are presented in figure 6 , with the three benchmark values of $g^{\prime} / \sin \theta=0.01,1$ and 10 respectively in the upper, middle and lower panels. The key difference between the $\mathrm{U}(1)_{B}$ and $\mathrm{U}(1)_{B-L}$ models is the $R$ factor relation in eq. (3.19). As a result, for $e+p$ scattering in the $\mathrm{U}(1)_{B-L}$ model, we have a disappearing P2 sensitivity only at

$$
m_{Z^{\prime}}=0.57 m_{Z}[51 \mathrm{GeV}]
$$

for $g^{\prime} / \sin \theta=0.01$, while for larger $g^{\prime}=1$ and 10 the factor $R$ is below -1 and there is no peak. Regarding $e+{ }^{12} \mathrm{C}$ scattering, $\mathrm{P} 2$ looses sensitivity at the following $Z^{\prime}$ masses:

$$
m_{Z^{\prime}}=1.06 m_{Z}[96 \mathrm{GeV}], \quad 3.57 m_{Z^{\prime}}[325 \mathrm{GeV}], \quad 10.9 m_{Z^{\prime}}[990 \mathrm{GeV}]
$$

for the three values of $g^{\prime} / \sin \theta=0.01,1$ and 10, respectively. The P2 sensitivities of $m_{Z^{\prime}}$ and $\sin \theta$ are also summarized in table 5 .

For the $\mathrm{U}(1)_{B}$ and $\mathrm{U}(1)_{B-L}$ models, the couplings of the $Z^{\prime}$ boson to proton and ${ }^{12} \mathrm{C}$ receive contributions from the direct coupling and the $Z-Z^{\prime}$ mixing, which are proportional to $g^{\prime}$ and $\sin \theta$, respectively. When the gauge coupling $g^{\prime}$ is sufficiently large, the couplings of $Z^{\prime}$ to proton and ${ }^{12} \mathrm{C}$ will be dominated by the direct coupling $g^{\prime}$, which is different from the pure $Z-Z^{\prime}$ mass mixing case in figure 3 . On the other hand, for sufficiently large $g^{\prime}$ and light $Z^{\prime}$, the new physics contribution to the asymmetry will be proportional to the $R$ factor, i.e. $\Delta A^{\mathrm{PV}} \propto R$ in eq. (3.16). Taking into account the different factors for proton and ${ }^{12} \mathrm{C}$ in eq. (4.5), for the cases of $g^{\prime} / \sin \theta=0.01,1$ and 10 for $\mathrm{U}(1)_{B}$ and the cases of $g^{\prime} / \sin \theta=1$ and 10 for $\mathrm{U}(1)_{B-L}$, the $\mathrm{P} 2$ sensitivities for low $m_{Z^{\prime}}$ are accidentally roughly the same for proton and ${ }^{12} \mathrm{C}$, as shown in figures 5 and 6.

\section{Conclusion}

With longitudinally polarized electron scattering off proton and ${ }^{12} \mathrm{C}$, the $\mathrm{P} 2$ experiment can perform a measurement of the parity-violating asymmetry $A^{\mathrm{PV}}$ at the unprecedented 

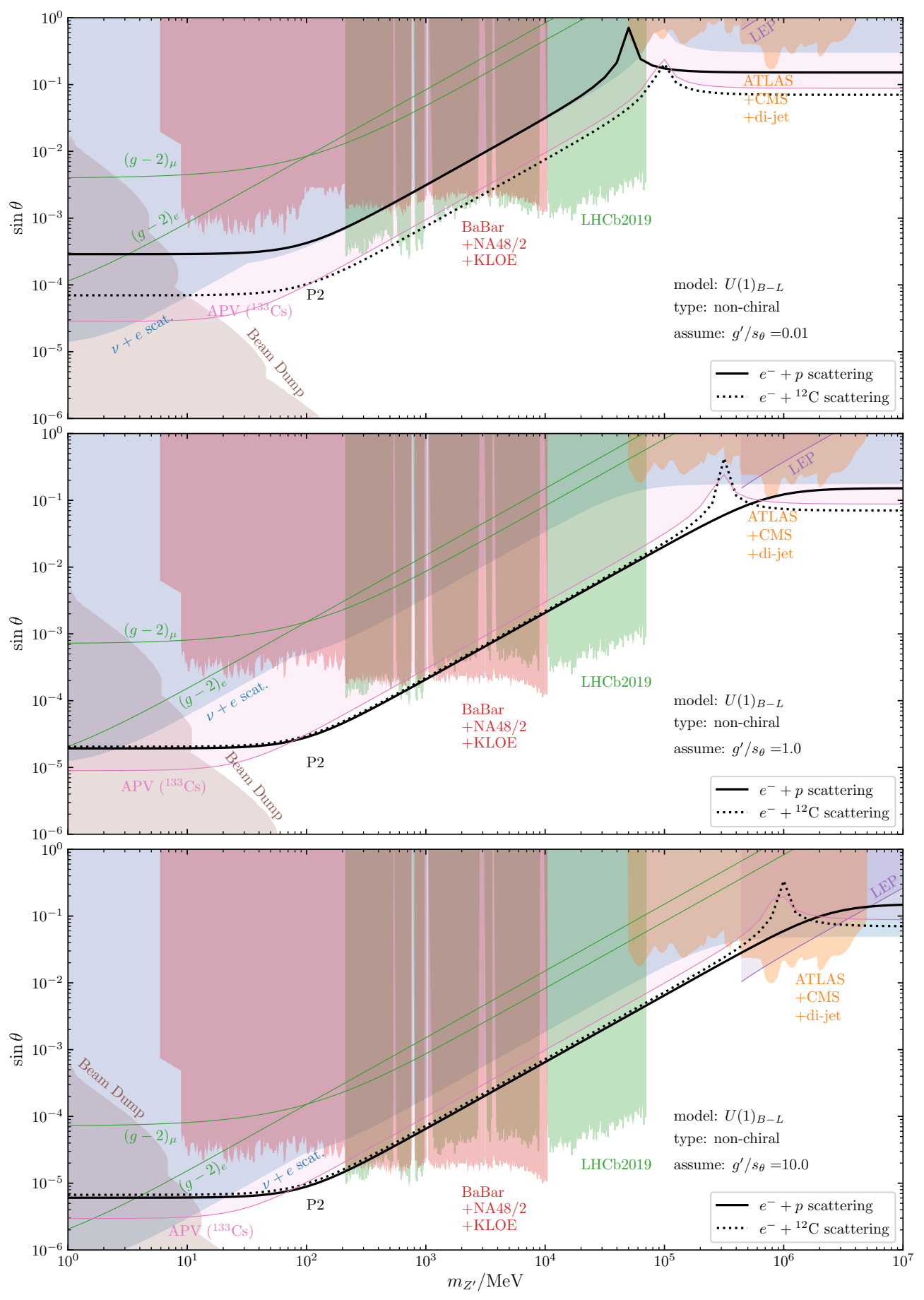

Figure 6. Similar to figure 5 but for the $\mathrm{U}(1)_{B-L}$ model. 
precision of below per cent level, which would result in a precision of $3.3 \times 10^{-4}$ for the weak mixing angle $s_{W}$ at sub-GeV scales. Such high-precision measurements have excellent sensitivities to potential BSM physics, as we have illustrated in this paper, taking as a case study arguably the most straightforward scenario: new $Z^{\prime}$ bosons. We assumed chiral and non-chiral $\mathrm{U}(1)^{\prime}$ models, for the latter case assuming kinetic or mass $Z-Z^{\prime}$ mixing. For the chiral case, where the $Z^{\prime}$ couplings to left- and right-handed fermions are different, we adopt three $\mathrm{U}(1)^{\prime}$ models, i.e. the $\mathrm{U}(1)_{L}^{\prime}, \mathrm{U}(1)_{R}^{\prime}$ and $\mathrm{U}(1)_{X}^{\prime}$ models given in table 2 . For the non-chiral models, we first consider a generic $\mathrm{U}(1)^{\prime}$ model with either mass $(\sin \theta)$ or kinetic $(\epsilon)$ mixing of $Z^{\prime}$ with the $Z$ boson and setting $g^{\prime}$ to zero, and then generalize to the $\mathrm{U}(1)_{B}$ and $\mathrm{U}(1)_{B-L}$ models with three different benchmark values of $g^{\prime} / \sin \theta=0.01$, 1 and 10.

For all chiral and non-chiral models, the leading-order new physics contributions are dominated by the interference terms of the BSM diagrams with the SM terms, i.e. terms proportional to the gauge couplings $g^{\prime 2}$, the mass mixing angle $\sin ^{2} \theta$ or the kinetic mixing parameter $\epsilon^{2}$. The $\mathrm{P} 2$ sensitivities for these $\mathrm{U}(1)^{\prime}$ models, as well as the current limits, are presented in figures 2 to 6 . Let us summarize our main results from these figures:

- It is a general feature that when the $Z^{\prime}$ mass is smaller than the energy scale $Q \simeq 93 \mathrm{MeV}$ of the P2 experiment, i.e. $m_{Z^{\prime}} \lesssim Q$, the P2 sensitivities will be independent of the $Z^{\prime}$ mass, as shown in the second and third columns of table 3 . However, the P2 prospects in the low $Z^{\prime}$ mass range are mostly precluded by the beam-dump experiments, and some are also limited by APV measurements, neutrino scattering data, electron and muon $g-2$, and electroweak precision data.

- In the large $Z^{\prime}$ mass limit, for the chiral $\mathrm{U}(1)^{\prime}$ models and the kinetic mixing case of non-chiral models, the P2 sensitivities are proportional to $g^{\prime} / m_{Z^{\prime}}$ or $\epsilon / m_{Z^{\prime}}$. In other words, the P2 experiment can probe effectively a UV cutoff scale $\Lambda=m_{Z^{\prime}} / g^{\prime}$ or $\Lambda=m_{Z^{\prime}} / \epsilon$. For the chiral models, the P2 experiment has sensitivities to a $Z^{\prime}$ mass up to $79 \mathrm{TeV}$ in the proton mode and up to $90 \mathrm{TeV}$ in ${ }^{12} \mathrm{C}$ mode, assuming the perturbative gauge coupling limit of $g^{\prime}=4 \pi$, as shown in table 4 . For the case of mass mixing $\sin \theta$ in non-chiral models, when the $Z^{\prime}$ boson is very heavy, say $m_{Z^{\prime}} \gtrsim \sqrt{1+R} m_{Z}$, the first term in the bracket of eq. (3.13) or (3.16) will be highly suppressed, and the $\mathrm{P} 2$ prospects for $\sin \theta$ will be independent of the $Z^{\prime}$ mass, which is very different from the cases above. The P2 sensitivities in the limit of heavy $Z^{\prime}$ bosons are collected in the fourth and fifth columns of table 3 .

One can see that for most of the cases in this paper, i.e. the three chiral models, generic $\mathrm{U}(1)^{\prime}$ with mass mixing $(\sin \theta)$, the $\mathrm{U}(1)_{B}$ model, and the $\mathrm{U}(1)_{B-L}$ model with $g^{\prime} / \sin \theta=0.01$ and 1 , the P2 experiment can probe a high UV scale $\Lambda$ or a mass mixing angle $\sin \theta$ that is currently not constrained and even goes beyond the direct search limits from LHC, in particular for $e+{ }^{12} \mathrm{C}$ scattering for most of the models. It is promising that a superheavy $Z^{\prime}$ boson can be directly searched for at future $100 \mathrm{TeV}$ colliders $[127,128]$, which is largely complementary to the prospects at the high-precision P2 experiment. The P2 prospects for a heavy $Z^{\prime}$ boson in the kinetic 
mixing $\mathrm{U}(1)^{\prime}$ model is precluded by electroweak precision data, while the $\mathrm{U}(1)_{B-L}$ model with a large gauge coupling $g^{\prime} / \sin \theta$ is excluded by neutrino scattering data for a heavy $Z^{\prime}$ boson.

- One may wonder whether the P2 experiment running with a ${ }^{12} \mathrm{C}$ target could have significantly improved sensitivity to new physics compared to that with a proton target. From figures 2 to 6 , we can see that this is rather model-dependent, subject to the quantum numbers of SM fermions under the $\mathrm{U}(1)^{\prime}$ gauge group for the chiral $\mathrm{U}(1)^{\prime}$ model, and depending on the gauge coupling $g^{\prime}$ for the non-chiral models. For the $\mathrm{U}(1)_{R}^{\prime}$ model, there is no sensitivity with the ${ }^{12} \mathrm{C}$ target, which is due to an accidental cancellation of the quantum numbers in eq. (3.5). For the $\mathrm{U}(1)_{L}^{\prime}$ model, the ${ }^{12} \mathrm{C}$ target can improve the sensitivity from the proton target by a factor of 1.4 , whereas for the $\mathrm{U}(1)_{X}^{\prime}$ model the prospects at proton and ${ }^{12} \mathrm{C}$ targets are roughly the same. For non-chiral models, the ${ }^{12} \mathrm{C}$ target does exhibit better sensitivity when $g^{\prime}$ is small; see figures 3,5 and 6 . In the limit of $g^{\prime}=0$, as shown in figures 3 , this improvement can reach a factor of 2 .

In any case, the comparison of electron-proton and electron- ${ }^{12} \mathrm{C}$ scatterings can obviously help in distinguishing models. Comparing further with the parity asymmetry in polarized electron-electron (Møller) scattering provides further handle on identifying the underlying physics [7].

- The nuclear $6 S_{1 / 2}-7 S_{1 / 2}$ transition in ${ }^{133} \mathrm{Cs}$ provides stringent limits on the chiral and non-chiral models which are comparable to the P2 prospects. Depending on model details, the P2 experiment can achieve better sensitivities than the APV measurements, in particular if the $Z^{\prime}$ boson is relatively heavy.

Taking into account all relevant existing constraints, we find that P2 can probe a broad range of $m_{Z^{\prime}}$ in the $\mathrm{U}(1)^{\prime}$ models considered in this paper, which are collected in table 4 for chiral models and table 5 for non-chiral models.

One should note that the specific $\mathrm{U}(1)^{\prime}$ models considered here are only for illustration purposes, and the analysis in this paper can be easily generalized to other $\mathrm{U}(1)^{\prime}$ models, by taking different choices of the quantum numbers $x$ and $y$ in eq. (3.1), or to flavor-sensitive scenarios such as the $L_{\mu}-L_{\tau}$ model.

\section{Acknowledgments}

We thank Niklaus Berger, Jeff Dror, Bhaskar Dutta and Krishna Kumar for helpful discussions. The work of B.D. is supported in part by the US Department of Energy under Grant No. DE-SC0017987, by the Neutrino Theory Network Program, and by a Fermilab Intensity Frontier Fellowship. X.J.X. is supported by the "Probing dark matter with neutrinos" ULB-ARC convention and by the F.R.S./FNRS under the Excellence of Science (EoS) project No. 30820817 - be.h "The $H$ boson gateway to physics beyond the Standard Model". Y.Z. is partially supported by "the Fundamental Research Funds for the Central Universities". 


\section{A The contribution of the axial-vector coupling}

In this appendix we show that the effect of $g_{A}$ (and $g_{A}^{\prime}$ ) is suppressed by a factor of $E_{e} / m_{N}$ (which in P2 is about 0.16 for electron-proton scattering, and 0.013 for electron${ }^{12} \mathrm{C}$ scattering) compared to the leading-order contribution to $A^{\mathrm{PV}}$ from $g_{V}$ (and $g_{V}^{\prime}$ ). To evaluate the contributions of $g_{A}$ and $g_{A}^{\prime}$, we first write down the full amplitude:

$$
\begin{aligned}
i \mathcal{M}_{L, R} \propto & {\left[\overline{u_{3}} \gamma^{\mu} P_{L, R} u_{1}\right]\left[\overline{u_{4}} \gamma_{\mu} u_{2}\right] \frac{-e^{2}}{q^{2}} } \\
& +\left[\overline{u_{3}} \gamma^{\mu} P_{L, R} g_{L, R} u_{1}\right]\left[\overline{u_{4}} \gamma_{\mu}\left(g_{V}+g_{A} \gamma^{5}\right) u_{2}\right] \frac{1}{q^{2}-m_{Z}^{2}} \\
& +\left[\overline{u_{3}} \gamma^{\mu} P_{L, R} g_{L, R}^{\prime} u_{1}\right]\left[\overline{u_{4}} \gamma_{\mu}\left(g_{V}^{\prime}+g_{A}^{\prime} \gamma^{5}\right) u_{2}\right] \frac{1}{q^{2}-m_{Z^{\prime}}^{2}}
\end{aligned}
$$

Hence eq. (2.4) is modified to

$$
i \mathcal{M}_{L, R} \propto\left[\overline{u_{3}} \gamma^{\mu} P_{L, R} u_{1}\right]\left[\overline{u_{4}} \gamma_{\mu}\left(G_{L, R}+G_{A} \gamma^{5}\right) u_{2}\right],
$$

where

$$
G_{A}=\frac{g_{L, R} g_{A}}{q^{2}-m_{Z}^{2}}+\frac{g_{L, R}^{\prime} g_{A}^{\prime}}{q^{2}-m_{Z^{\prime}}^{2}} .
$$

Applying the standard trace technology, we obtain:

$$
\begin{aligned}
\left|\mathcal{M}_{L, R}\right|^{2} \propto & G_{L, R}^{2}\left(2-2 y-4 r_{2} y-r_{1}^{2}+4 r_{2}^{2} y^{2}\right)-8 G_{L, R} G_{A}\left(1-r_{2} y\right) r_{2} y \\
& +G_{A}^{2}\left(2+2 y-4 r_{2} y+r_{1}^{2}+4 r_{2}^{2} y^{2}\right)
\end{aligned}
$$

where $r_{1} \equiv m_{e} / E_{e} \approx 3 \times 10^{-3}, r_{2} \equiv E_{e} / m_{N} \approx 0.16$ (for $e+p$ scattering) or 0.013 (for $e+{ }^{12} \mathrm{C}$ scattering), and $y \equiv-q^{2} / 4 E_{e}^{2} \approx 0.09$. The second term in eq. (A.4) proportional to $G_{A}$ implies that the axial-vector contribution is suppressed by $r_{2} y$. The third term proportional to $G_{A}^{2}$, though not suppressed, cancels in $\left|\mathcal{M}_{R}\right|^{2}-\left|\mathcal{M}_{L}\right|^{2}$.

Open Access. This article is distributed under the terms of the Creative Commons Attribution License (CC-BY 4.0), which permits any use, distribution and reproduction in any medium, provided the original author(s) and source are credited.

\section{References}

[1] Particle Data Group collaboration, Review of Particle Physics, PTEP 2020 (2020) 083C01 [INSPIRE].

[2] M.S. Safronova, D. Budker, D. DeMille, D.F.J. Kimball, A. Derevianko and C.W. Clark, Search for New Physics with Atoms and Molecules, Rev. Mod. Phys. 90 (2018) 025008 [arXiv: 1710.01833] [INSPIRE].

[3] J. Erler, C.J. Horowitz, S. Mantry and P.A. Souder, Weak Polarized Electron Scattering, Ann. Rev. Nucl. Part. Sci. 64 (2014) 269 [arXiv:1401.6199] [InSPIRE].

[4] P. Souder and K.D. Paschke, Parity violation in electron scattering, Front. Phys. (Beijing) 11 (2016) 111301 [INSPIRE]. 
[5] SLAC E158 collaboration, Observation of parity nonconservation in Møller scattering, Phys. Rev. Lett. 92 (2004) 181602 [hep-ex/0312035] [INSPIRE].

[6] SLAC E158 collaboration, Precision measurement of the weak mixing angle in Møller scattering, Phys. Rev. Lett. 95 (2005) 081601 [hep-ex/0504049] [INSPIRE].

[7] MOLLER collaboration, The MOLLER Experiment: An Ultra-Precise Measurement of the Weak Mixing Angle Using Møller Scattering, arXiv:1411.4088 [INSPIRE].

[8] SAMPLE collaboration, Parity violation in elastic electron proton scattering and the proton's strange magnetic form-factor, Phys. Rev. Lett. 84 (2000) 1106 [nucl-ex/9909010] [INSPIRE].

[9] HAPPEX collaboration, New measurement of parity violation in elastic electron - proton scattering and implications for strange form-factors, Phys. Lett. B 509 (2001) 211 [nucl-ex/0006002] [INSPIRE].

[10] HAPPEX collaboration, Parity violating electroweak asymmetry in polarized-e $p$ scattering, Phys. Rev. C 69 (2004) 065501 [nucl-ex/0402004] [INSPIRE].

[11] G0 collaboration, The G0 Experiment: Apparatus for Parity-Violating Electron Scattering Measurements at Forward and Backward Angles, Nucl. Instrum. Meth. A 646 (2011) 59 [arXiv:1103.0761] [INSPIRE].

[12] Qweak collaboration, Precision measurement of the weak charge of the proton, Nature $\mathbf{5 5 7}$ (2018) 207 [arXiv: 1905. 08283] [inSPIRE].

[13] D. Becker et al., The P2 experiment, Eur. Phys. J. A 54 (2018) 208 [arXiv:1802.04759] [INSPIRE].

[14] C.Y. Prescott et al., Parity Nonconservation in Inelastic Electron Scattering, Phys. Lett. B 77 (1978) 347 [INSPIRE].

[15] C.Y. Prescott et al., Further Measurements of Parity Nonconservation in Inelastic electron Scattering, Phys. Lett. B 84 (1979) 524 [INSPIRE].

[16] SAMPLE collaboration, Parity violating electron deuteron scattering and the proton's neutral weak axial vector form-factor, Phys. Rev. Lett. 92 (2004) 102003 [nucl-ex/0310001] [INSPIRE].

[17] PVDIS collaboration, Measurement of parity violation in electron-quark scattering, Nature 506 (2014) 67 [INSPIRE].

[18] D. Wang et al., Measurement of Parity-Violating Asymmetry in Electron-Deuteron Inelastic Scattering, Phys. Rev. C 91 (2015) 045506 [arXiv:1411.3200] [INSPIRE].

[19] D. Balaguer Ríos et al., Measurement of the parity violating asymmetry in the quasielastic electron-deuteron scattering and improved determination of the magnetic strange form factor and the isovector anapole radiative correction, Phys. Rev. D 94 (2016) 051101 [INSPIRE].

[20] HAPPEX collaboration, Parity-violating electron scattering from He-4 and the strange electric form-factor of the nucleon, Phys. Rev. Lett. 96 (2006) 022003 [nucl-ex/0506010] [INSPIRE].

[21] W. Heil et al., Improved Limits on the Weak, Neutral, Hadronic Axial Vector Coupling Constants From Quasielastic Scattering of Polarized Electrons, Nucl. Phys. B 327 (1989) 1 [INSPIRE]. 
[22] P.A. Souder et al., Measurement of parity violation in the elastic scattering of polarized electrons from C-12, Phys. Rev. Lett. 65 (1990) 694 [INSPIRE].

[23] S. Abrahamyan et al., Measurement of the Neutron Radius of 208Pb Through Parity-Violation in Electron Scattering, Phys. Rev. Lett. 108 (2012) 112502 [arXiv:1201.2568] [INSPIRE].

[24] C.S. Wood et al., Measurement of parity nonconservation and an anapole moment in cesium, Science 275 (1997) 1759.

[25] S.C. Bennett and C.E. Wieman, Measurement of the $6 S \rightarrow 7 S$ transition polarizability in atomic cesium and an improved test of the Standard Model, Phys. Rev. Lett. 82 (1999) 2484 [Erratum ibid. 82 (1999) 4153] [Erratum ibid. 83 (1999) 889] [hep-ex/9903022] [INSPIRE].

[26] S.G. Porsev, K. Beloy and A. Derevianko, Precision determination of electroweak coupling from atomic parity violation and implications for particle physics, Phys. Rev. Lett. 102 (2009) 181601 [arXiv:0902.0335] [INSPIRE].

[27] S.G. Porsev, K. Beloy and A. Derevianko, Precision determination of weak charge of ${ }^{133} \mathrm{Cs}$ from atomic parity violation, Phys. Rev. D 82 (2010) 036008 [arXiv:1006.4193] [inSPIRE].

[28] N.H. Edwards, S.J. Phipp, P.E.G. Baird and S. Nakayama, Precise Measurement of Parity Nonconserving Optical Rotation in Atomic Thallium, Phys. Rev. Lett. 74 (1995) 2654 [INSPIRE].

[29] P.A. Vetter, D.M. Meekhof, P.K. Majumder, S.K. Lamoreaux and E.N. Fortson, Precise test of electroweak theory from a new measurement of parity nonconservation in atomic thallium, Phys. Rev. Lett. 74 (1995) 2658 [INSPIRE].

[30] D.M. Meekhof, P. Vetter, P.K. Majumder, S.K. Lamoreaux and E.N. Fortson, High-precision measurement of parity nonconserving optical rotation in atomic lead, Phys. Rev. Lett. 71 (1993) 3442 [INSPIRE].

[31] M.J.D. Macpherson, K.P. Zetie, R.B. Warrington, D.N. Stacey and J.P. Hoare, Precise measurement of parity nonconserving optical rotation at $876 \mathrm{~nm}$ in atomic bismuth, Phys. Rev. Lett. 67 (1991) 2784 [INSPIRE].

[32] D. Antypas, A. Fabricant, J.E. Stalnaker, K. Tsigutkin, V.V. Flambaum and D. Budker, Isotopic variation of parity violation in atomic ytterbium, Nature Phys. 15 (2019) 120 [arXiv: 1804.05747] [INSPIRE].

[33] W.J. Marciano and J.L. Rosner, Atomic parity violation as a probe of new physics, Phys. Rev. Lett. 65 (1990) 2963 [Erratum ibid. 68 (1992) 898] [INSPIRE].

[34] G. Altarelli, R. Casalbuoni, S. De Curtis, N. Di Bartolomeo, F. Feruglio and R. Gatto, Atomic parity violation in extended gauge models and latest CDF and LEP data, Phys. Lett. B 261 (1991) 146 [INSPIRE].

[35] J. Erler, A. Kurylov and M.J. Ramsey-Musolf, The Weak charge of the proton and new physics, Phys. Rev. D 68 (2003) 016006 [hep-ph/0302149] [INSPIRE].

[36] A. Kurylov, M.J. Ramsey-Musolf and S. Su, Probing supersymmetry with parity violating electron scattering, Phys. Rev. D 68 (2003) 035008 [hep-ph/0303026] [INSPIRE].

[37] J. Erler, P. Langacker, S. Munir and E. Rojas, Z' Bosons from $E_{6}$ : Collider and Electroweak Constraints, in 19th International Workshop on Deep-Inelastic Scattering and Related Subjects, (2011) [arXiv:1108.0685] [INSPIRE]. 
[38] R. Diener, S. Godfrey and I. Turan, Constraining Extra Neutral Gauge Bosons with Atomic Parity Violation Measurements, Phys. Rev. D 86 (2012) 115017 [arXiv:1111.4566] [INSPIRE].

[39] H. Davoudiasl, H.-S. Lee and W.J. Marciano, Muon Anomaly and Dark Parity Violation, Phys. Rev. Lett. 109 (2012) 031802 [arXiv:1205.2709] [INSPIRE].

[40] H. Davoudiasl, H.-S. Lee and W.J. Marciano, Muon g - 2, rare kaon decays, and parity violation from dark bosons, Phys. Rev. D 89 (2014) 095006 [arXiv:1402.3620] [InSPIRE].

[41] P.S.B. Dev, M.J. Ramsey-Musolf and Y. Zhang, Doubly-Charged Scalars in the Type-II Seesaw Mechanism: Fundamental Symmetry Tests and High-Energy Searches, Phys. Rev. D 98 (2018) 055013 [arXiv: 1806.08499] [INSPIRE].

[42] H.N. Long, N.V. Hop, L.T. Hue and N.T.T. Van, Constraining heavy neutral gauge boson $Z^{\prime}$ in the 3-3-1 models by weak charge data of Cesium and proton, Nucl. Phys. B 943 (2019) 114629 [arXiv: 1812.08669] [inSPIRE].

[43] R.D. Carlini, W.T.H. van Oers, M.L. Pitt and G.R. Smith, Determination of the Proton's Weak Charge and Its Constraints on the Standard Model, Ann. Rev. Nucl. Part. Sci. 69 (2019) 191 [INSPIRE].

[44] G. D'Ambrosio, A.M. Iyer, F. Piccinini and A.D. Polosa, Confronting B anomalies with low energy parity violation, Phys. Rev. D 101 (2020) 035025 [arXiv: 1902.00893] [InSPIRE].

[45] G. Arcadi, M. Lindner, J. Martins and F.S. Queiroz, New physics probes: Atomic parity violation, polarized electron scattering and neutrino-nucleus coherent scattering, Nucl. Phys. B 959 (2020) 115158 [arXiv: 1906.04755] [INSPIRE].

[46] M. Ghosh, Y. Grossman and W. Tangarife, Probing the two-neutrino exchange force using atomic parity violation, Phys. Rev. D 101 (2020) 116006 [arXiv:1912.09444] [INSPIRE].

[47] D.K. Hong, Parity violation and new physics in superconductors, Phys. Lett. B 811 (2020) 135950 [arXiv: 2009.01494].

[48] B.K. Sahoo, B.P. Das and H. Spiesberger, New Physics Constraints from Atomic Parity Violation in ${ }^{133} \mathrm{Cs}$, arXiv:2101.10095 [INSPIRE].

[49] S. Oda, N. Okada and D.-s. Takahashi, Classically conformal U(1)' extended standard model and Higgs vacuum stability, Phys. Rev. D 92 (2015) 015026 [arXiv:1504.06291] [INSPIRE].

[50] M.D. Campos, D. Cogollo, M. Lindner, T. Melo, F.S. Queiroz and W. Rodejohann, Neutrino Masses and Absence of Flavor Changing Interactions in the 2HDM from Gauge Principles, JHEP 08 (2017) 092 [arXiv: 1705. 05388] [INSPIRE].

[51] I. Alikhanov and E.A. Paschos, A chiral gauge-invariant model for Majorana neutrinos, arXiv: 1902.09950 [INSPIRE].

[52] B. Dutta, S. Ghosh and J. Kumar, A sub-GeV dark matter model, Phys. Rev. D 100 (2019) 075028 [arXiv: 1905.02692] [INSPIRE].

[53] S. Jana, P.K. Vishnu and S. Saad, Minimal Dirac neutrino mass models from U(1) $)_{\mathrm{R}}$ gauge symmetry and left-right asymmetry at colliders, Eur. Phys. J. C 79 (2019) 916 [arXiv: 1904.07407] [INSPIRE].

[54] R.H. Helm, Inelastic and Elastic Scattering of 187-Mev Electrons from Selected Even-Even Nuclei, Phys. Rev. 104 (1956) 1466 [INSPIRE]. 
[55] A. Davidson, $B-L$ as the fourth color within an $\mathrm{SU}(2)_{L} \times \mathrm{U}(1)_{R} \times \mathrm{U}(1)$ model, Phys. Rev. D 20 (1979) 776 [INSPIRE].

[56] R.N. Mohapatra and R.E. Marshak, Local B-L Symmetry of Electroweak Interactions, Majorana Neutrinos and Neutron Oscillations, Phys. Rev. Lett. 44 (1980) 1316 [Erratum ibid. 44 (1980) 1643] [INSPIRE].

[57] W. Buchmüller, C. Greub and P. Minkowski, Neutrino masses, neutral vector bosons and the scale of B-L breaking, Phys. Lett. B 267 (1991) 395 [INSPIRE].

[58] M. Lindner, F.S. Queiroz, W. Rodejohann and X.-J. Xu, Neutrino-electron scattering: general constraints on $Z^{\prime}$ and dark photon models, JHEP 05 (2018) 098 [arXiv: 1803.00060] [INSPIRE].

[59] R. Foot, G.C. Joshi and H. Lew, Gauged Baryon and Lepton Numbers, Phys. Rev. D 40 (1989) 2487 [INSPIRE].

[60] R. Kempf, J. Diefenbach, F. Fichtner and K. Aulenbacher, Beam parameter stabilization for the P2 experiment at MESA, Nucl. Instrum. Meth. A 982 (2020) 164554.

[61] J. Erler and M.J. Ramsey-Musolf, The Weak mixing angle at low energies, Phys. Rev. D 72 (2005) 073003 [hep-ph/0409169] [INSPIRE].

[62] J. Erler and R. Ferro-Hernández, Weak Mixing Angle in the Thomson Limit, JHEP 03 (2018) 196 [arXiv: 1712.09146] [INSPIRE].

[63] K.S. Kumar, S. Mantry, W.J. Marciano and P.A. Souder, Low Energy Measurements of the Weak Mixing Angle, Ann. Rev. Nucl. Part. Sci. 63 (2013) 237 [arXiv:1302.6263] [INSPIRE].

[64] R. Essig et al., Working Group Report: New Light Weakly Coupled Particles, in Community Summer Study 2013: Snowmass on the Mississippi, (2013) [arXiv:1311.0029] [INSPIRE].

[65] P. Ilten, Y. Soreq, M. Williams and W. Xue, Serendipity in dark photon searches, JHEP 06 (2018) 004 [arXiv: 1801.04847] [INSPIRE].

[66] M. Bauer, P. Foldenauer and J. Jaeckel, Hunting All the Hidden Photons, JHEP 07 (2018) 094 [arXiv: 1803.05466] [inSPIRE].

[67] J.M. Berryman, A. de Gouvêa, P.J. Fox, B.J. Kayser, K.J. Kelly and J.L. Raaf, Searches for Decays of New Particles in the DUNE Multi-Purpose Near Detector, JHEP 02 (2020) 174 [arXiv: 1912.07622] [INSPIRE].

[68] M. Fabbrichesi, E. Gabrielli and G. Lanfranchi, The Dark Photon, arXiv:2005.01515 [INSPIRE].

[69] G. Lanfranchi, M. Pospelov and P. Schuster, The Search for Feebly-Interacting Particles, arXiv: 2011.02157 [INSPIRE].

[70] W.J. Marciano and A. Sirlin, Radiative corrections to atomic parity violation, Phys. Rev. D 27 (1983) 552 [INSPIRE].

[71] W.J. Marciano and A. Sirlin, On Some General Properties of the O(alpha) Corrections to Parity Violation in Atoms, Phys. Rev. D 29 (1984) 75 [Erratum ibid. 31 (1985) 213] [INSPIRE].

[72] H. Davoudiasl, H.-S. Lee and W.J. Marciano, 'Dark' Z implications for Parity Violation, Rare Meson Decays, and Higgs Physics, Phys. Rev. D 85 (2012) 115019 [arXiv:1203.2947] [INSPIRE]. 
[73] M. Abdullah, J.B. Dent, B. Dutta, G.L. Kane, S. Liao and L.E. Strigari, Coherent elastic neutrino nucleus scattering as a probe of a $Z$ ' through kinetic and mass mixing effects, Phys. Rev. D 98 (2018) 015005 [arXiv: 1803.01224] [InSPIRE].

[74] J.D. Bjorken et al., Search for Neutral Metastable Penetrating Particles Produced in the SLAC Beam Dump, Phys. Rev. D 38 (1988) 3375 [inSPIRE].

[75] E.M. Riordan et al., A Search for Short Lived Axions in an Electron Beam Dump Experiment, Phys. Rev. Lett. 59 (1987) 755 [InSPIRE].

[76] A. Bross, M. Crisler, S.H. Pordes, J. Volk, S. Errede and J. Wrbanek, A Search for Shortlived Particles Produced in an Electron Beam Dump, Phys. Rev. Lett. 67 (1991) 2942 [INSPIRE].

[77] M. Davier and H. Nguyen Ngoc, An Unambiguous Search for a Light Higgs Boson, Phys. Lett. B 229 (1989) 150 [INSPIRE].

[78] NA64 collaboration, Constraints on New Physics in the Electron g-2 from a Search for Invisible Decays of a Scalar, Pseudoscalar, Vector, and Axial Vector, Phys. Rev. Lett. 126 (2021) 21 [arXiv:2102.01885] [INSPIRE].

[79] BABAR collaboration, Search for a Dark Photon in $e^{+} e^{-}$Collisions at BaBar, Phys. Rev. Lett. 113 (2014) 201801 [arXiv: 1406 . 2980] [INSPIRE].

[80] BaBar collaboration, Search for Invisible Decays of a Dark Photon Produced in $e^{+} e^{-}$ Collisions at BaBar, Phys. Rev. Lett. 119 (2017) 131804 [arXiv:1702. 03327] [InSPIRE].

[81] NA48/2 collaboration, Search for the dark photon in $\pi^{0}$ decays, Phys. Lett. B 746 (2015) 178 [arXiv: 1504.00607] [INSPIRE].

[82] KLOE-2 collaboration, Limit on the production of a light vector gauge boson in $\phi$ meson decays with the KLOE detector, Phys. Lett. B $\mathbf{7 2 0}$ (2013) 111 [arXiv:1210.3927] [INSPIRE].

[83] P.J. Fox, R. Harnik, J. Kopp and Y. Tsai, LEP Shines Light on Dark Matter, Phys. Rev. D 84 (2011) 014028 [arXiv: 1103.0240] [InSPIRE].

[84] LHCb collaboration, Search for $A^{\prime} \rightarrow \mu^{+} \mu^{-}$Decays, Phys. Rev. Lett. 124 (2020) 041801 [arXiv: 1910.06926] [INSPIRE].

[85] ATLAS collaboration, Search for high-mass dilepton resonances using $139 \mathrm{fb}^{-1}$ of $\mathrm{pp}$ collision data collected at $\sqrt{s}=13 \mathrm{TeV}$ with the ATLAS detector, Phys. Lett. B 796 (2019) 68 [arXiv: 1903.06248] [INSPIRE].

[86] ATLAS collaboration, Search for New Phenomena in Dijet Events using $139 \mathrm{fb}^{-1}$ of pp collisions at $\sqrt{s}=13 \mathrm{TeV}$ collected with the ATLAS Detector, ATLAS-CONF-2019-007.

[87] CMS collaboration, Search for a narrow resonance in high-mass dilepton final states in proton-proton collisions using $140 \mathrm{fb}^{-1}$ of data at $\sqrt{s}=13 \mathrm{TeV}$, CMS-PAS-EXO-19-019.

[88] CMS collaboration, Search for high mass dijet resonances with a new background prediction method in proton-proton collisions at $\sqrt{s}=13 \mathrm{TeV}$, JHEP 05 (2020) 033 [arXiv: 1911.03947] [INSPIRE].

[89] DELPHI collaboration, Measurement and interpretation of fermion-pair production at LEP energies above the $Z$ resonance, Eur. Phys. J. C 45 (2006) 589 [hep-ex/0512012] [INSPIRE].

[90] E. Bagnaschi et al., Global Analysis of Dark Matter Simplified Models with Leptophobic Spin-One Mediators using MasterCode, Eur. Phys. J. C 79 (2019) 895 [arXiv: 1905. 00892] [INSPIRE]. 
[91] E.J. Chun, A. Das, J. Kim and J. Kim, Searching for flavored gauge bosons, JHEP 02 (2019) 093 [Erratum ibid. 07 (2019) 024] [arXiv: 1811.04320] [INSPIRE].

[92] CHARM-II collaboration, Measurement of differential cross-sections for muon-neutrino electron scattering, Phys. Lett. B 302 (1993) 351 [INSPIRE].

[93] CHARM-II collaboration, Precision measurement of electroweak parameters from the scattering of muon-neutrinos on electrons, Phys. Lett. B 335 (1994) 246 [INSPIRE].

[94] LSND collaboration, Measurement of electron - neutrino - electron elastic scattering, Phys. Rev. D 63 (2001) 112001 [hep-ex/0101039] [INSPIRE].

[95] G. Bellini et al., Precision measurement of the 7Be solar neutrino interaction rate in Borexino, Phys. Rev. Lett. 107 (2011) 141302 [arXiv:1104.1816] [InSPIRE].

[96] TEXONO collaboration, Measurement of $\bar{\nu}_{e}$-Electron Scattering Cross-Section with a CsI(Tl) Scintillating Crystal Array at the Kuo-Sheng Nuclear Power Reactor, Phys. Rev. D 81 (2010) 072001 [arXiv:0911.1597] [INSPIRE].

[97] COHERENT collaboration, Observation of Coherent Elastic Neutrino-Nucleus Scattering, Science 357 (2017) 1123 [arXiv: 1708.01294] [INSPIRE].

[98] S. Bilmis, I. Turan, T.M. Aliev, M. Deniz, L. Singh and H.T. Wong, Constraints on Dark Photon from Neutrino-Electron Scattering Experiments, Phys. Rev. D 92 (2015) 033009 [arXiv: 1502.07763] [INSPIRE].

[99] Y. Farzan, M. Lindner, W. Rodejohann and X.-J. Xu, Probing neutrino coupling to a light scalar with coherent neutrino scattering, JHEP 05 (2018) 066 [arXiv:1802.05171] [INSPIRE].

[100] J.P. Leveille, The Second Order Weak Correction to (G-2) of the Muon in Arbitrary Gauge Models, Nucl. Phys. B 137 (1978) 63 [InSPIRE].

[101] T. Aoyama et al., The anomalous magnetic moment of the muon in the Standard Model, Phys. Rept. 887 (2020) 1 [arXiv:2006.04822] [INSPIRE].

[102] H. Davoudiasl and W.J. Marciano, Tale of two anomalies, Phys. Rev. D 98 (2018) 075011 [arXiv: 1806.10252] [INSPIRE].

[103] T. Aoyama, T. Kinoshita and M. Nio, Revised and Improved Value of the QED Tenth-Order Electron Anomalous Magnetic Moment, Phys. Rev. D 97 (2018) 036001 [arXiv: 1712.06060] [INSPIRE].

[104] R.H. Parker, C. Yu, W. Zhong, B. Estey and H. Müller, Measurement of the fine-structure constant as a test of the Standard Model, Science 360 (2018) 191 [arXiv:1812.04130] [INSPIRE].

[105] L. Morel, Z. Yao, P. Cladé and S. Guellati-Khélifa, Determination of the fine-structure constant with an accuracy of 81 parts per trillion, Nature 588 (2020) 61 [INSPIRE].

[106] M. Lindner, M. Platscher and F.S. Queiroz, A Call for New Physics: The Muon Anomalous Magnetic Moment and Lepton Flavor Violation, Phys. Rept. 731 (2018) 1

[arXiv:1610.06587] [INSPIRE].

[107] D. Curtin, R. Essig, S. Gori and J. Shelton, Illuminating Dark Photons with High-Energy Colliders, JHEP 02 (2015) 157 [arXiv: 1412.0018] [INSPIRE].

[108] M. Pospelov, Secluded U(1) below the weak scale, Phys. Rev. D 80 (2009) 095002 [arXiv:0811.1030] [INSPIRE]. 
[109] J.A. Dror, R. Lasenby and M. Pospelov, New constraints on light vectors coupled to anomalous currents, Phys. Rev. Lett. 119 (2017) 141803 [arXiv:1705.06726] [INSPIRE].

[110] J.A. Dror, R. Lasenby and M. Pospelov, Dark forces coupled to nonconserved currents, Phys. Rev. D 96 (2017) 075036 [arXiv:1707.01503] [INSPIRE].

[111] J.A. Dror, R. Lasenby and M. Pospelov, Light vectors coupled to bosonic currents, Phys. Rev. D 99 (2019) 055016 [arXiv: 1811.00595] [INSPIRE].

[112] C.D. Carone and H. Murayama, Possible light U(1) gauge boson coupled to baryon number, Phys. Rev. Lett. 74 (1995) 3122 [hep-ph/9411256] [INSPIRE].

[113] P. Fayet, Constraints on Light Dark Matter and $U$ bosons, from $\psi, \Upsilon, K^{+}, \pi^{\circ}, \eta$ and $\eta^{\prime}$ decays, Phys. Rev. D 74 (2006) 054034 [hep-ph/0607318] [INSPIRE].

[114] P. Fayet, U-boson production in $e^{+} e^{-}$annihilations, $\psi$ and $\Upsilon$ decays, and Light Dark Matter, Phys. Rev. D 75 (2007) 115017 [hep-ph/0702176] [INSPIRE].

[115] BaBAR collaboration, Search for Production of Invisible Final States in Single-Photon Decays of $\Upsilon(1 S)$, Phys. Rev. Lett. 107 (2011) 021804 [arXiv:1007.4646] [INSPIRE].

[116] CLEO collaboration, Search for Very Light CP-Odd Higgs Boson in Radiative Decays of $\Upsilon(S-1)$, Phys. Rev. Lett. 101 (2008) 151802 [arXiv:0807.1427] [INSPIRE].

[117] L3 collaboration, Search for new physics in energetic single photon production in $e^{+} e^{-}$ annihilation at the $Z$ resonance, Phys. Lett. B 412 (1997) 201 [INSPIRE].

[118] DELPHI collaboration, Search for neutral heavy leptons produced in $Z$ decays, Z. Phys. C 74 (1997) 57 [Erratum ibid. 75 (1997) 580] [INSPIRE].

[119] L3 collaboration, Isolated hard photon emission in hadronic $Z^{0}$ decays, Phys. Lett. B 292 (1992) 472 [INSPIRE].

[120] OPAL collaboration, A Measurement of photon radiation in lepton pair events from $Z^{0}$ decays, Phys. Lett. B 273 (1991) 338 [INSPIRE].

[121] L3 collaboration, Search for narrow high mass resonances in radiative decays of the $Z^{0}$, Phys. Lett. B 262 (1991) 155 [INSPIRE].

[122] E. Rrapaj and S. Reddy, Nucleon-nucleon bremsstrahlung of dark gauge bosons and revised supernova constraints, Phys. Rev. C 94 (2016) 045805 [arXiv:1511.09136] [InSPIRE].

[123] J.H. Chang, R. Essig and S.D. McDermott, Revisiting Supernova 1987A Constraints on Dark Photons, JHEP 01 (2017) 107 [arXiv: 1611.03864] [INSPIRE].

[124] A. Fradette, M. Pospelov, J. Pradler and A. Ritz, Cosmological Constraints on Very Dark Photons, Phys. Rev. D 90 (2014) 035022 [arXiv: 1407.0993] [InSPIRE].

[125] M. Escudero, D. Hooper, G. Krnjaic and M. Pierre, Cosmology with A Very Light $L_{\mu}-L_{\tau}$ Gauge Boson, JHEP 03 (2019) 071 [arXiv:1901.02010] [INSPIRE].

[126] S. Knapen, T. Lin and K.M. Zurek, Light Dark Matter: Models and Constraints, Phys. Rev. D 96 (2017) 115021 [arXiv: 1709.07882] [INSPIRE].

[127] N. Arkani-Hamed, T. Han, M. Mangano and L.-T. Wang, Physics opportunities of a $100 \mathrm{TeV}$ proton-proton collider, Phys. Rept. 652 (2016) 1 [arXiv:1511.06495] [INSPIRE].

[128] T. Golling et al., Physics at a $100 \mathrm{TeV}$ pp collider: beyond the Standard Model phenomena, arXiv: 1606.00947 [INSPIRE]. 